\title{
Generative Adversarial Networks for Hyperspectral Image Classification
}

\author{
Lin Zhu, Yushi Chen, Member, IEEE, Pedram Ghamisi, Member, IEEE, and Jón Atli Benediktsson,
}

Fellow, IEEE

\begin{abstract}
A Generative Adversarial Network (GAN) usually contains a generative network and a discriminative network in competition with each other. The GAN has shown their capability in a variety of applications. In this paper, the usefulness and effectiveness of GAN for classification of hyperspectral images (HSIs) is explored for the first time. In the proposed GAN, a convolutional neural network (CNN) is designed to discriminate the inputs and another $\mathrm{CNN}$ is used to generate so-called fake inputs. The aforementioned CNNs are trained together: The generative $\mathrm{CNN}$ tries to generate fake inputs that are as real as possible, and the discriminative $\mathrm{CNN}$ tries to classify the real and fake inputs. This kind of adversarial training improves the generalization capability of the discriminative $\mathrm{CNN}$, which is really important when the training samples are limited. Specifically, we propose two schemes: (1) a well-designed 1D-GAN as a spectral classifier, and (2) a robust 3D-GAN is as a spectral-spatial classifier. Furthermore, the generated adversarial samples are used with real training samples to fine-tune the discriminative $\mathrm{CNN}$, which improves the final classification performance. The proposed classifiers are carried out on three widely-used hyperspectral datasets: Salinas, Indiana Pines, and Kennedy Space Center. The obtained results reveal that the proposed models provide competitive results compared to the state-of-the-art methods. In addition, the proposed GANs open new opportunities in the remote sensing community for the challenging task of HSIs classification, and also, reveal the huge potential of GAN-based methods for the analysis of such complex and inherently nonlinear data.
\end{abstract}

Index Terms - Convolutional neural network (CNN), deep learning, generative adversarial network (GAN), hyperspectral images classification.

\section{INTRODUCTION}

$\mathrm{H}$ YPERSPECTRAL sensors simultaneously capture the

This work was supported in part by the Natural Science Foundation of China under the Grant 61771171 and in part by the National Natural Science Foundation of key international cooperation under the Grant 61720106002. (Corresponding author: Yushi Chen)

L. Zhu and Y. Chen are with the School of Electronics and Information Engineering, Harbin Institute of Technology, Harbin 150001, China (e-mail: 17s105139@stu.hit.edu.cn; chenyushi@hit.edu.cn).

P. Ghamisi is with German Aerospace Center (DLR), Remote Sensing Technology Institute (IMF), Wessling 82234, Germany, and also with the Signal Processing in Earth Observation (SiPEO), Technical University of Munich (TUM), Munich 80333, Germany (e-mail: p.ghamisi@gmail. com).

J. A. Benediktsson is with the Faculty of Electrical and Computer Engineering, University of Iceland, IS-107 Reykjavik, Iceland (e-mail: benedikt@hi.is) spatial and spectral information of the observing target. Such data are characterized as a valuable source of information for Earth observation, which brings both challenges and opportunities to develop new data processing techniques [1]. The classification of hyperspectral images (HSIs), which is a crucial step for the plethora of applications, tries to assign a specific class to each pixel in the scene. HSIs classification is widely-used, e.g. urban development, land change monitoring, scene interpretation, and resource management. Among hyperspectral data processing techniques, classification is one of the most vibrant topics in the remote sensing community [2].

Most of the supervised methods that have been applied in the machine learning community have been explored for hyperspectral images classification in the last few decades. In general, there are two kinds of data classification methods: spectral classifiers and spectral-spatial classifiers.

Hyperspectral data usually contain hundreds of spectral channels of the same scene, which provide abundant spectral information. Traditional spectral classification algorithms typically include k-nearest-neighbors, maximum likelihood, neural network and logistic regression [3]. Most of those algorithms dramatically suffer from the so-called curse of dimensionality (Hughes phenomenon) [4]. Support vector machines (SVMs) have shown their low sensitivity to high dimensionality with respect to the number of training samples and are unlikely to suffer from the Hughes phenomenon. Furthermore, SVM-based classifiers usually obtain good classification performance in terms of when a limited number of training samples is available compared with other widely-used supervised techniques [5] [6]. During the first decade of this century, SVMs-based spectral classifiers were considered as the state-of-the-art methods in the hyperspectral community.

With the advancement of the sensor and imaging systems, the spatial resolution of hyperspectral data is becoming finer and finer. With the help of spatial information, which can be extracted by diverse methodologies such as filtering or segmentation approaches, the classification performance can be significantly improved. Among those approaches, morphological profiles have been widely-used to extract the spatial features of HSIs (usually followed by SVMs or random forest to obtain the resulting classification map) [7]. Many extensions have been developed for hyperspectral data feature extraction based on morphological operators, which demonstrate that the morphological profile is still under further 
development [8]. On the other hand, multiple kernel learning has been applied to hyperspectral data classification because of its powerful capability to handle heterogeneous spectral and spatial features in an efficient manner [9]. Spectral-spatial classification approaches are regarded as the mainstream of HSI classification. Many methods have been proposed in recent years on the spectral-spatial classification of hyperspectrla data. As an example, in [10], a method, which combines sparse representation and Markov random fields, was proposed. The method utilizes Markov random fields to explore spatial correlation which significantly improves the classification performance. Furthermore, a new spectral-spatial classifier based on discriminant analysis has been proposed for hyperspectral image classification in [11]. The proposed method learned a representative subspace from the spectral and spatial domains and produced a good classification performance.

Most of the traditional spectral and spectral-spatial classifiers do not classify the hyperspectral data in a "deep" manner. In [12], it is demonstrated that the classifiers like linear SVM and logistic regression can be attributed to single-layer classifiers, while decision tree or SVM with kernels are believed to have two layers. Deep models, which contain two or more hidden layers, tend to extract the invariant and discriminant features of the input data. Deep learning methods have been actively studied in a wide variety of research areas such as image classification, language processing, and speech recognition in recent years [13] [14]. Recently, deep learning has also been utilized for remote sensing data processing including HSI classification. A survey of deep learning-based approaches for remote sensing data can be found in [15].

In 2014, a deep learning-based method, stacked autoencoders, has been proposed for hyperspectral feature extraction and classification [16]. Deep belief network, another kind of deep models, was introduced and modified for HSI classification as well [17] [18].

In recent years, lots of deep models, especially deep convolutional neural networks (CNNs), have been proposed in the remote sensing community. In [19], a deep CNN with five layers was employed to extract the spectral features of HSIs leading to a promising classification performance. After that, in [20], a novel pixel-pair method is proposed as a deep spectral classifier, and the model achieved good performance when the number of training samples is not sufficient.

The aforementioned methods in [19] and [20] are spectral classifiers. In order to utilize the spatial and spectral information of HSIs simultaneously, some spectral-spatial classifiers based on deep CNNs have been proposed [21], [22]. For example, in [22] a framework based on principal component analysis (PCA), deep CNN, and logistic regression was used for HSIs classification. HSIs are inherently 3D data, so it is reasonable to design 3D CNNs to effectively extract the spectral-spatial features of HSIs [23], [24]. On the other hand, CNNs can be combined with other techniques such as sparse representation approach and morphological profiles. In [25], $\mathrm{CNN}$ is followed by sparse representation to refine the learned features. Recently, a method based on the combination of
Gabor filtering and $\mathrm{CNN}$ was introduced to extract the features of HSIs, which leads to a performance improvement [26].

Furthermore, there are new types of deep models which have also been investigated for HSIs classification. Very recently, a new deep dictionary learning has been proposed for HSIs classification, which has shown its capability under the condition of limited training samples [27].

Although great progress has been achieved in HSI classification using deep learning-based methods, deep models usually face a serious problem known as overfitting. The reason behind this problem is that deep learning-based methods need to train a large number of learnable parameters, which requires a lot of training samples. The problem becomes serious when the number of training samples is limited. Unfortunately, the limited availability of training samples is a common situation in the remote sensing community since the collection of such data is either time demanding or expensive. Deep models are often over trained if there are not sufficient training samples available, which means the performance is good in the training stage but the performance is relatively poor in the test stage. Therefore, new and effective training strategies for deep models are needed to address the issue of overfitting (the Generative Adversarial Network (GAN), which this paper investigates, is one of those strategies). Actually, the generator network of GAN can be regarded as a regularization method, which can mitigate the overfitting phenomena to a great extent.

GAN is a new kind of model, which usually contains agenerative model $G$ and a discriminative model $D$ [28]. The models $G$ and $D$ are trained in an adversarial manner, in which $G$ tries to generate the fake inputs as real as possible, and $D$ tries to classify the real and fake inputs. In this adversarial game, both participants wish to get optimized results (i.e., $D$ can achieve the best classification results, and $G$ can generate the fake data which possess the most similar distribution with real data). Through the adversarial manner and competition of two networks, the training process of the discriminator will proceed both continuously and effectively instead of getting trapped into overfitting immediately when we use a limited number of training samples.

Furthermore, GAN generates samples which can be used as virtual samples. The proper usage of the virtual samples improves the classification performance. In this paper, the generated samples are used to boost the classification accuracy and the experimental results prove the effectiveness of the usage of such samples.

Due to the advantages of CNN, deep convolutional GAN is well suited for image data processing [29]. GAN can be used in a variety of applications such as data synthesis, style transfer, image super-resolution and classification [30].

In this paper, the use of the generative adversarial network for hyperspectral data classification is explored for the first time. With the help of GANs, the deep CNN achieves better performance in terms of classification accuracy and the overfitting problem raised by CNNs can be considerably mitigated. Two frameworks, 1D-GAN and 3D-GAN, are proposed for HSI classification, and the classification results obtained by these two frameworks showed that our GANs are 


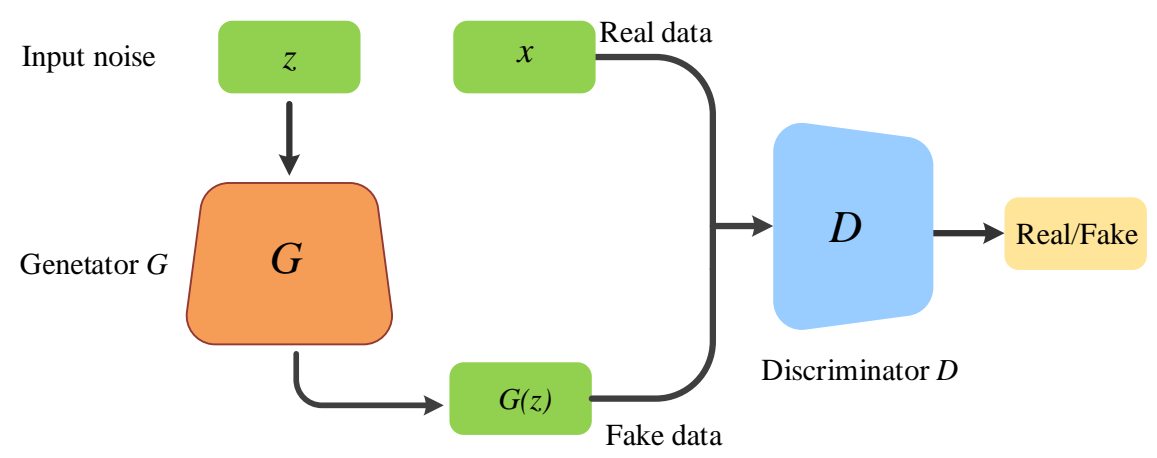

Fig. 1. The architecture of GAN

superior to traditional convolution neural networks even under the condition of limited training samples. In more details, the main contributions of the paper are summarized as follows.

1) The use of GAN for hyperspectral data processing is explored for the first time in this paper. The adversarial samples including 1D spectra and 3D spectral-spatial patches are generated by a well-designed GAN.

2) Two GANs frameworks including spectral classifiers and spectral-spatial classifiers are proposed for HSIs classification. The frameworks use well-designed CNNs to classify HSIs, and the adversarial training is adopted by a regularization technique.

3) Adversarial samples are used for HSIs classification for the first time, which is investigated to fine-tune the aforementioned GANs for an improved classification performance.

4) The proposed methods are tested on three well-known hyperspectral datasets under the condition of having limited training samples available.

The rest of the paper is organized as follows. Section II presents the background of GAN. Section III presents the details of the proposed GAN frameworks including spectral and spectral-spatial architectures for HSIs classification, and the introduction of adversarial samples for classification. The details of experimental results are reported in Section IV. In Section V, conclusions and discussions are presented.

\section{BACKGROUND}

Supervised learning methods in machine learning can in general be divided into two parts: Generative approaches and discriminate approaches. Generative approaches involve the learning of distribution parameters from data samples, and they can generate new samples according to the learned models. Typically, generative methods calculate the distribution assumption of the explicit or implicit variables of the real data. Then, they can generate new data through the learned assumptions, which have a similar distribution as the real data.

GAN which was proposed by Goodfellow in 2014, is a novel way to train a generative mode land a promising method to train classifiers. Commonly GAN is composed of two parts: the generative network $G$ and the discriminative model $D$.The generator $G$ can capture the potential distribution of real data and output the new data while the discriminator $D$ is a binary classifier which can judge whether the input samples are real or not. The information flow in GAN is a feed forward pass from one model generator $G$, which generates the fake data, to the second model discriminator $D$, which evaluates the output of the first model. The architecture of GAN is shown in Fig. 1.

In order to learn the generator's distribution $p_{g}$ over data $x$, we supposed that the true samples are equipped with data distribution $p(x)$ and the input noise variable has a prior $p(z)$ The generator accepts a random noise $z$ as input and produces a mapping to data space $G(z)$.The $D(x)$ estimates the probability that $x$ is the true sample from training data [31]. In the optimized procedure the discriminator $D$ is trained to maximize $\log (D(x))$ which is the probability of assigning the correct labels to the correct sources while the generator $G$ is trained to minimize $\log (1-D(G(z)))$. Therefore, the ultimate aim of the optimization is to solve the minimax problem:

$$
\begin{aligned}
\min _{G} \max _{D} V(D, G) & \left.=\mathrm{E}_{x \sim p(x)} \mid \log (D(x))\right] \\
& +\mathrm{E}_{z \sim p(z)}[\log (1-D(G(z)))]
\end{aligned}
$$

where $E$ is the expectation operator. Through the calculation and evaluation, one found that when the discriminator $D$ has a high probability distribution of real samples, the gradients in $D$ may disappear and cause the training process to stop. To make sure that the generator has proper gradients when the classification accuracy for the discriminator is high, the generator's loss function is usually formulated to maximize the probability of classifying a sample as true instead of minimizing the probability of it to be classified as false [30]. Therefore, the modified loss function can be written in the following optimization form:

$$
\begin{aligned}
& \begin{aligned}
F(D, G) & =\min _{D} V(D, G) \\
& =-\mathrm{E}_{x \sim p(x)}[\log (D(x))]-\mathrm{E}_{z \sim p(z)}[\log (1-D(G(z)))] \\
f(D, G) & =\min _{G} V(D, G)=-\mathrm{E}_{z \sim p(z)}[\log (D(G(z)))]
\end{aligned}
\end{aligned}
$$

The parameters update of $G$ is based on the back-propagation of $D$ instead of directly using the real data samples. In addition, 


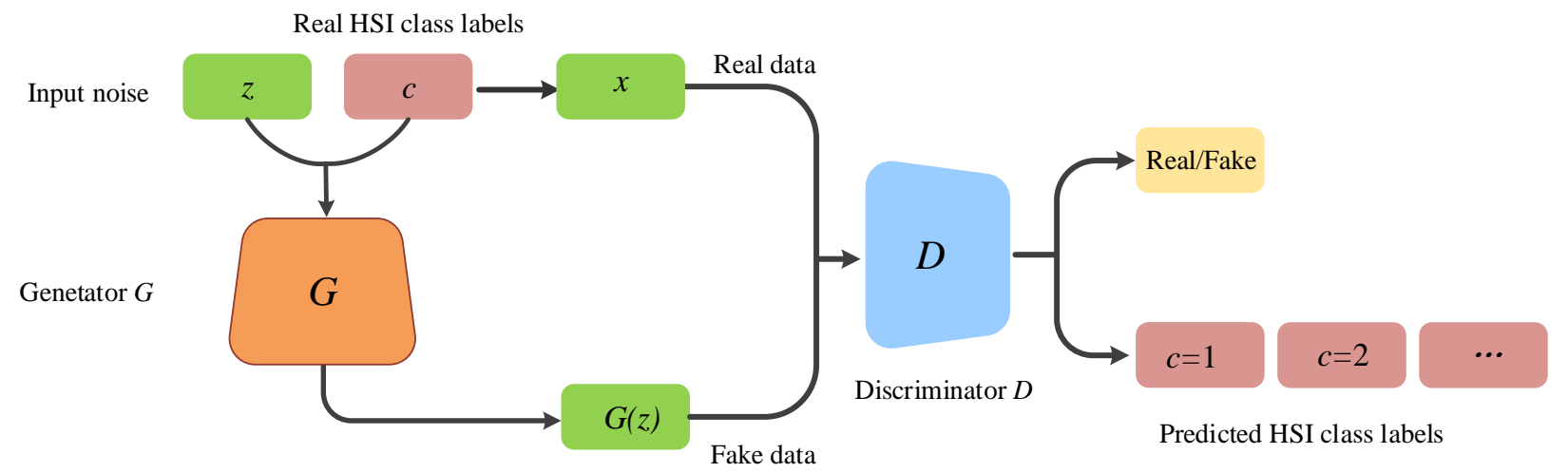

Fig. 2. The general architecture of proposed method

there is no requirement that the latent code has any specific dimensionality nor on the generator net being invertible. GANs can learn models that generate points only on a thin manifold that goes near the data, so the GAN framework can train any kind of generator net.

On one hand, the adversarial model is most straightforward when the $G$ and $D$ are both multiply perceptrons [28]. On the other hand, the shallow multiply perceptrons usually have limited capabilities in coping with complex data. Recently, deep learning methods have achieved a substantial success and they can build a network with several layers instead of having shallow net layer connections [33]. Therefore, they can leverage the nonlinear mapping abilities and capture more crucial features of input data (i.e., especially in coping with the complicated and inherently nonlinear data) [34] [35]. As a special type of deep learning-based method, the convolution network (i.e., CNN) [36], which is inspired by neuroscience [37], utilizes two important strategies which make it different from other deep learning methods. Those two strategies are local connections and shared weights. In more details, in the $\mathrm{CNN}$, the local correlation information is explored by considering the local connectivity between nearby neurons, instead of the full connection of all neural units as in other deep methods. In this context, CNNs can reduce the computational complexity and redundancy in exploiting the data features. Especially, CNNs can alleviate the overfitting phenomenon in the deep learning process. Recently, the deep methods and CNNs have shown their excellent performance in hyperspectral images classification and feature extraction [23].

Although GANs have shown their promising usages in varieties of aspects, they also suffer from stability issues due to its adversarial idea [28]. To solve this issue, some techniques for stabilizing the training process of the GAN have been developed. Among those approaches, the convolution network can address the instability issue to some extent. Recently, the deep convolutional generative adversarial network (DCGAN) architecture, which uses deep convolution networks in $G$ and $D$ was proposed [29]. The generator $G$, which takes a uniform noise distribution $z$ and class labels $c$ as input and output, respectively, can be reshaped into a three-dimensional tensor. The discriminator $D$ has a similar architecture to common CNNs except that the pooling layers are replaced with stride convolutions and all the activation functions are the leaky rectified linear unit (LeakyReLU) [38]. Although GANs have shown promising performance in image synthesis [39] and many other aspects [40], as unsupervised generative models, the $D$ networks of the original GANs only estimate whether the input samples are either true or fake. Therefore, they are not suitable for the purpose of multi-class image classification. Recently, the concept of GAN has been extended to be a conditional model with semi-supervised methods. In [41], the authors proposed Semi-GAN whose labels of true training data were imported to the discriminator $D$.

Furthermore, the conditional GAN requires some additional information for both $G$ and $D$ where the extra information can be class labels or other data modalities [42]. Therefore, the conditional GAN can leverage the generative adversarial nets to generate the specific labels. In addition, Odena presented auxiliary classifier GAN (AC-GAN), which can be used in image classifications, whose discriminator $D$ is modified to be a softmax classifier that can output multi-class labels probabilities [43].

\section{PROPOSED METHOD}

\section{A. The framework of the proposed method}

Our networks are shaped with respect to the theory of AC-GAN while the objective function is modified to take into account the likelihood of the correct source and likelihood of the correct HSI classes. The parameters are optimized depending on the multi-classification loss so they can optimize the loss function more appropriately than traditional GANs. The labeled information is regarded as the input of both generator and discriminator, and the discriminator $D$ contains an auxiliary decoder network which can output the respective training data labels. Definitely, the extra-label information can leverage both the classification abilities of discriminator $D$ and generative abilities of generator $G$. The architecture of our method is shown in the Fig. 2.

From the original GAN, we can see that in training process, the discriminator $D$ receives both real training data and fake data generated by the generator $G$ and outputs the probability distribution $P(S \mid X)=D(X)$. Therefore, the aim of the $D$ 


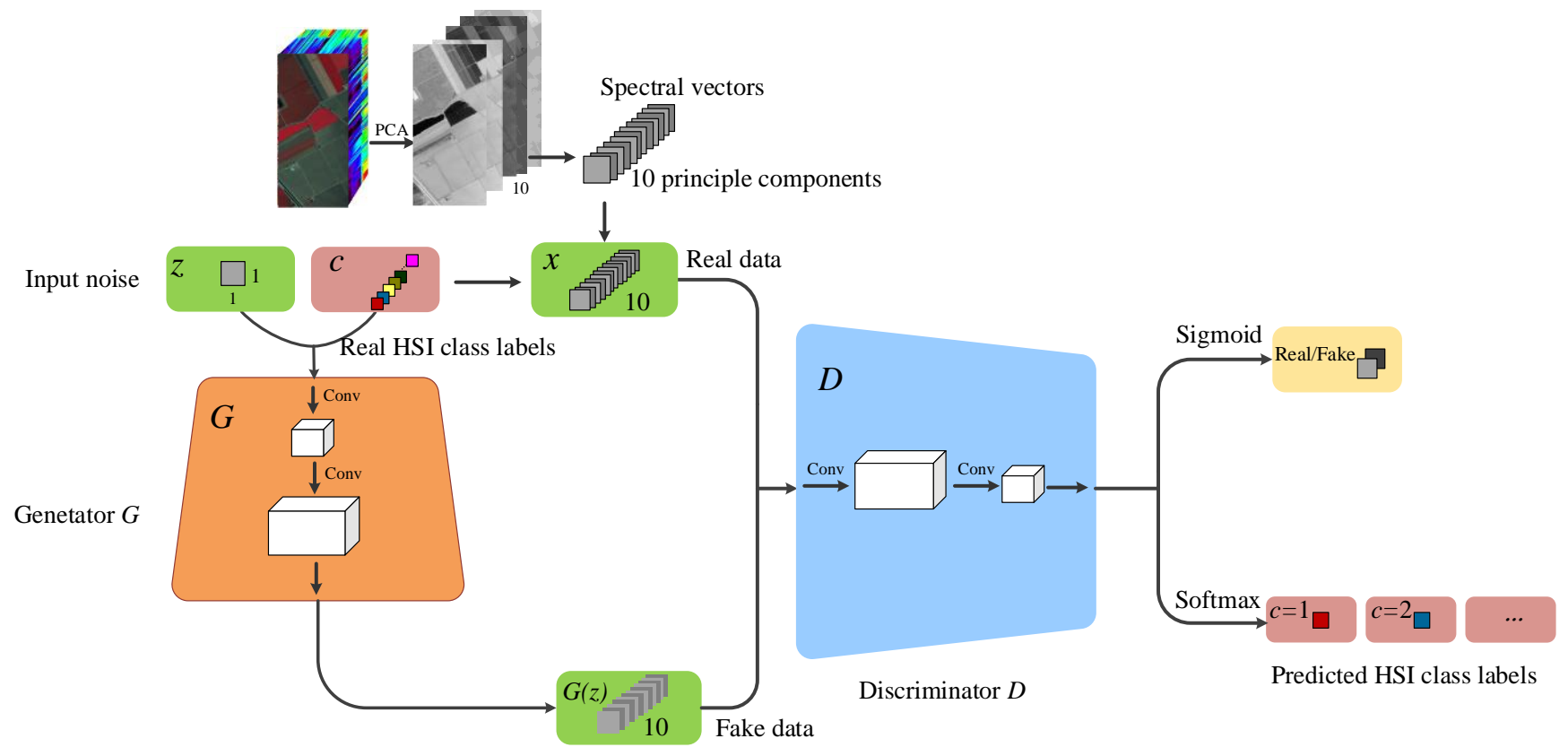

Fig. 3. The framework of 1D-GAN for HSIs classification

network is to try to maximize the log-likelihood of the right source:

$$
\begin{aligned}
L & =\mathrm{E}\left[\log P\left(S=\text { real } \mid X_{\text {real }}\right)\right] \\
& +\mathrm{E}\left[\log P\left(S=\text { fake } \mid X_{\text {fake }}\right)\right]
\end{aligned}
$$

Similarly, the aim of the $G$ network is to try to minimize the same quantity.

In the network, we can see that the generator $G$ also accept hyperspectral image class labels $c \sim p_{c}$ in addition to the noise $z$ the output of $G$ can be defined by $X_{\text {fake }}=G(z)$. The real training data with corresponding class labels and the fake data generated by $G$ are regarded as the input of the discriminator $D$, The probability distribution over sources $P(S \mid X)$ along with the probability distribution over class labels $P(C \mid X)$ are fed into the network $D$. The objective function contains two parts: the $\log$-likelihood of the right source of input data $L_{\mathrm{S}}$ and the $\log$-likelihood of the right class labels $L_{c}$ :

$$
\begin{aligned}
L_{s} & =\mathrm{E}\left[\log P\left(S=\text { real } \mid X_{\text {real }}\right)\right] \\
& +\mathrm{E}\left[\log P\left(S=\text { fake } \mid X_{\text {fake }}\right)\right] \\
L_{c} & =\mathrm{E}\left[\log P\left(C=c \mid X_{\text {real }}\right)\right] \\
& +\mathrm{E}\left[\log P\left(C=c \mid X_{\text {fake }}\right)\right]
\end{aligned}
$$

so $D$ is optimized to maximize the $L_{S}+L_{C}$ while $G$ is optimized to maximize $L_{C}-L_{S}$

In this experiment, the two frameworks (i.e., the combination of the spectral vector and the spectral-spatial information) are designed, while the former is called 1D-GAN and the latter is called 3D-GAN. The $G$ and $D$ in the aforementioned two frameworks are both in the form of convolutional networks. The proposed GANs are not only used to classify the real and fake inputs but also to predict the corresponding labels of the input data. $G$ has the form of fractionally-stride convolutional neural network and $D$ adopts convolution neural networks. Finally, the discriminator $D$ is followed by sigmoid classifier and softmax classifiers applied in parallel, which are used to classify the real/fake samples and the HSIs, respectively.

With the continuation of the training process, both $G$ and $D$ will theoretically achieve the most optimized results when $G$ can generate fake data that are most similar to real data and $D$ can-not distinguish between fake data and real data. In this manner, we can prove that the whole network achieves the Nash equilibrium condition, and the adversarial behavior and competition between two networks can promote the classification performance. Therefore, the crucial idea of GAN lies in the adversarial training and through the continuous competition we can obtain superior classification results in comparison to traditional CNN methods.

\section{B. $1 D-G A N$}

The main framework of 1D-GAN is shown in Fig. 3, which is built based on HSI spectral features only where all the input noise and training data are spectral vectors.

Due to the availability of hundreds of bands in original HSI, which means the input has high dimensions, it is difficult to 


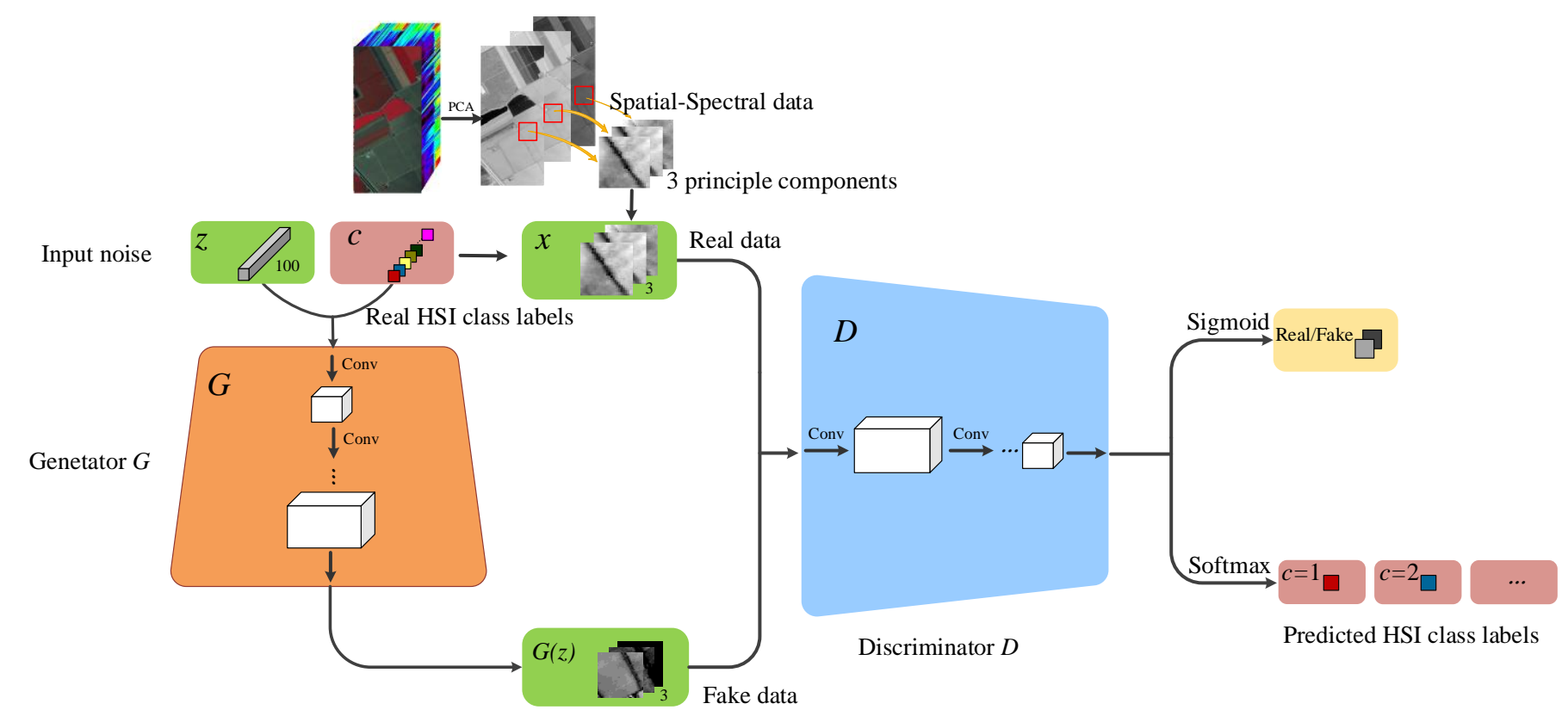

Fig. 4. The framework of 3D-GAN for HSIs classification

train the generator $G$ (i.e., the training stage is not stable). In the other words, the generator cannot effectively imitate the real data because of high redundancy. Thus, we extract the spectral features of HSIs based on principal component analysis (PCA) [44]. PCA can condense the whole image by reducing the spectral dimensions to a suitable scale, and it is a crucial step to formulate a robust GAN.

Pertinently, in order to promote the generator to exert superior abilities on capturing crucial features and generating realistic-looking samples as the best possible and preserve most important spectral information of original HSI bands, we preserve 10 principal components, which contain the $99.99 \%$ energy of the inputs.

In Fig. 3, we show the framework of 1D-GAN on the Salinas dataset as input. From the figure, one can see that the $G$ receives both noise $z$ and labels $c$ as input, while $D$ receives as input the real spectral vectors $x$ with labels $c$ and some batches of fake spectral vectors $G(z)$. Then, through the $D$ network, one can obtain the final results including both the class labels and the source labels. In our networks, any pooling layer is replaced with stride convolutions (discriminator) and fractional-strided convolutions (generator).

\section{C. $3 D-G A N$}

The main framework of 3D-GAN is shown in Fig.4. In 3D-GAN, the network extracts spectral-spatial features effectively, which achieves better classification performance. Here, we only describe the special parts of the proposed 3D-GAN. Different from 1D-GAN which only utilizes the spectral information of HSIs, the spatial features are also taken into consideration in addition to the spectral features in this subsection.

The number of spectral bands is reduced to three components by PCA, which condense the whole image by reducing the data dimensions to a suitable scale, and in the meantime, it reserves the spatial information. Due to PCA, the computational complexity is dramatically reduced, and it is really important to stabilize the GAN training procedure. In 3D-GAN, the generator accepts noise as input and transforms its shape to the same size as real data with three principal components in the spectral domain. Then, the discriminator accepts the real data or the generated fake samples as input data, and it uses the sigmoid classifier to give the real and fake classification results and softmax classifier to give the classification map. The first layer of the GAN, which takes a uniform noise distribution $z$ as input, is just a matrix multiplication. The result is then reshaped into a tensor and used as the start of the convolution stack. In the proposed frameworks, we indicate the hyperspectral ground labels in multicolor squares but the source labels in grey squares.

In 3D-GAN, like the basic allocations in 1D-GAN, pooling layers are replaced with strided convolutions (in the discriminator) and fractional-strided convolutions (in the generator). In addition, batch normalization is used in both the generator and the discriminator and the fully connected hidden layers are removed.

\section{Generative Adversarial Samples for Classification}

Now let us consider that the GAN consists of both a generator and a discriminator. The classification abilities of the discriminator have been discussed above. On the other hand, using the fake samples from the generator also has a potential in improving the final classification results in terms of accuracies. In fact, when the discriminator cannot distinguish the real data from the synthetic fake data, we can also conclude that the generated abilities of $G$ are superior to the $D$. Consequently, the whole adversarial network achieves the global optimality in 
theory [28].

In this paper, the generated fake samples can be regarded as augmentation data [45] that increase the number of training samples. Then, both the fake samples and the true samples are fed into the networks in order to optimize the nets. Let us suppose that the original dataset has $N$ classes, during network training. To begin with, each generated sample is passed forward through the network and assigned a label by taking the maximum value of the probability prediction vector. These fake samples can consequently be used for training in the network with these labels. In addition, the generated samples do not belong to any class of real samples. Due to the difference of real samples and generated samples, a new class label is then created (i.e., $N+1$ ) and every fake sample is endowed with this new label. In this paper, we adopted this method to assign $N+1$ to the labels of fake samples. In the experimental part of the paper, we qualitatively visualize the adversarial samples through the false color map.

\section{EXPERIMENTAL RESULTS}

\section{A. Data Description}

In our study, three widely-used hyperspectral datasets with different environmental settings were adopted to validate the proposed methods. They are captured over Salinas Valley in California (Salinas), Kennedy Space Center (KSC) in Florida and a mixed vegetation site over the Indian Pines test area in North-western Indiana (Indian Pines).

The first dataset, Salinas, was collected by the 224-band AVIRIS sensor over Salinas Valley, California. The available dataset is composed of $512 \times 217$ pixels with 204 bands [after the removal of low signal to noise ratio (SNR) bands] and the available ground reference map covers 16 classes of interest. The hyperspectral image is characterized by having a high spatial resolution (3.7-meter pixels). The false color composite image and the corresponding ground reference map are demonstrated in Fig. 5.

The second dataset was captured by the NASA airborne

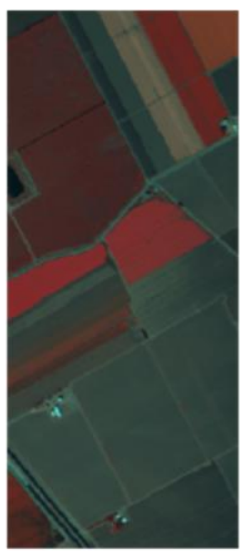

(a)

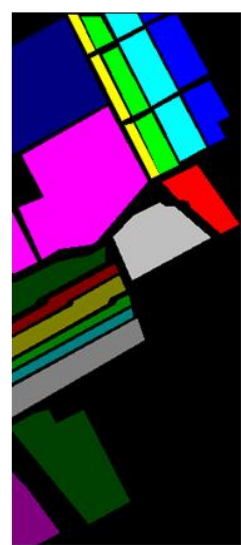

(b)
Fig. 5. The Salinas dataset. (a) False-color composite (Band 50, 28, 15) and (b) ground reference map
AVIRIS instrument over the Kennedy Space Center, Florida. It is called here the KSC dataset. The KSC dataset has an altitude of approximately $20 \mathrm{~km}$, with a spatial resolution of $18 \mathrm{~m}$. This dataset is composed of $512 \times 614$ pixels. After removing water absorption and low SNR bands, 176 bands were used for the corresponding false color composite map. In the classification, 13 classes were defined for the site. Fig. 6 demonstrates the classes of the KSC dataset and the corresponding false color composite map.

The third dataset is a mixed vegetation site over the Indian Pines test area in Northwestern Indiana (Indian Pines) which was collected by the Airborne Visible/Infrared Imaging Spectrometer (AVIRIS). The dataset was obtained by an aircraft flown, with a size of 145 pixels $\times 145$ pixels and 220 spectral bands in the wavelength range of $0.4-2.5 \mu \mathrm{m}$. The false color image is shown in Fig. 7(a). The number of bands is reduced to 200 by removing water absorption bands. Sixteen different land-cover classes are provided in the ground reference map, as shown in Fig. 7.

For all three datasets, the labeled samples were split into two subsets which contain training and test samples, and the details are listed in Table I, II, and III. During the training procedure of GAN, we use 200 training samples, which are truly limited, to learn weights and biases of each neuron, 100 training samples are used to guide the design of proper architectures, which can identify whether the network is overfitted or not. In the test process, all the samples in the dataset are used to estimate the capability of the trained network. This is important for designing the proper convolution network and we can use the test samples to assess the final classification performance.

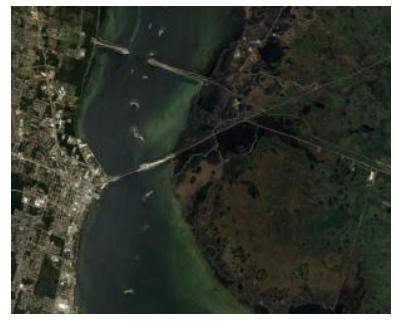

(a)

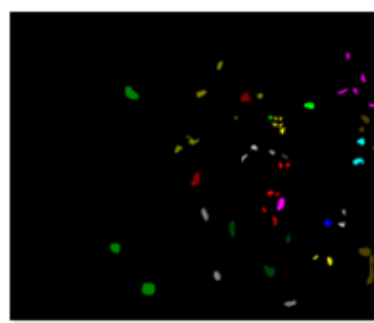

(b)
Fig. 6. The Kennedy Space Center (KSC) dataset. (a) False-color composite (Band 28, 19, 10) and (b) ground reference map

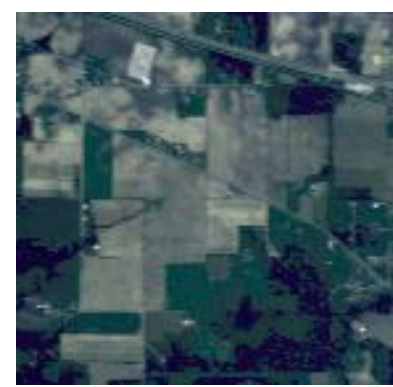

(a)

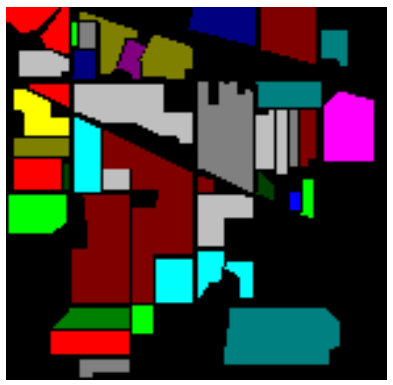

(b)
Fig. 7. The Indian Pines dataset. (a) False-color composite image (Band 28, 19, 10) and (b) ground reference map 
TABLE I LAND COVER Classes AND NUMBERS OF PIXELS ON SALINAS DATASET

\begin{tabular}{c|c|c|c}
\hline \hline \multicolumn{2}{|c|}{ Class } & Samples \\
\hline No & \multirow{2}{*}{ Color } & Name & Numbers \\
\hline 1 & & Brocoli_green_weeds_1 & 1977 \\
2 & & Brocoli_green_weeds_2 & 3726 \\
3 & & Fallow & 1976 \\
4 & & Fallow_rough_plow & 1394 \\
5 & & Fallow_smooth & 2678 \\
6 & & Stubble & 3959 \\
7 & & Celery & 3579 \\
8 & & Grapes_untrained & 11213 \\
9 & & Soil_vinyard_develop & 6197 \\
10 & & Corn_senesced_green_weeds & 3249 \\
11 & & Lettuce_romaine_4wk & 1058 \\
12 & & Lettuce_romaine_5wk & 1908 \\
13 & & Lettuce_romaine_6wk & 909 \\
14 & & Lettuce_romaine_7wk & 1061 \\
15 & & Vinyard_untrained & 7164 \\
16 & & Vinyard_vertical_trellis & 1737 \\
\hline \hline \multicolumn{3}{|c}{} & Total \\
\hline
\end{tabular}

TABLE II LAND COVER Classes AND NuMBERS OF PIXELS IN KSC DATASET

\begin{tabular}{c|c|c|c}
\hline \hline \multicolumn{2}{|c|}{ Class } & Samples \\
\hline No. & Color & Name & Numbers \\
\hline 1 & & Scrub & 761 \\
2 & & Willow swamp & 243 \\
3 & & CP hammock & 256 \\
4 & & Slash pine & 252 \\
5 & & Oak/Broadleaf & 161 \\
6 & & Hardwood & 229 \\
7 & & Swamp & 105 \\
8 & & Graminoid marsh & 431 \\
9 & & Spartina marsh & 520 \\
10 & & Cattail marsh & 404 \\
11 & & Salt marsh & 419 \\
12 & & Mud flats & 503 \\
13 & & Water & 927 \\
\hline \multicolumn{3}{|c|}{} \\
\hline \hline
\end{tabular}

\section{B. The classification results for $1 D-G A N$}

In this experiment, our networks are also compared with other former methods used in HSI feature extraction and classifications such as the original CNN to validate the quality of classification results. The training and test data are randomly chosen among the whole dataset and we only use 200 training samples for each datasets and the epoch is set to 500 for our methods. The GANs have a relatively higher computational complexity compared with other networks considering the fact that both the $G$ and $D$ need to be trained in each epoch. The classification results are given in the form of mean \pm standard deviation. The 1D-GAN framework is built only based on HSI
TABLE III LAND COVER Classes AND NuMBERS OF PIXELS ON INDIAN PINES DATASET

\begin{tabular}{|c|c|c|c|}
\hline \multicolumn{3}{|c|}{ Class } & \multirow{2}{*}{$\begin{array}{c}\text { Samples } \\
\text { Numbers }\end{array}$} \\
\hline No. & Color & Name & \\
\hline 1 & & Alfalfa & 46 \\
\hline 2 & & Corn-notill & 1428 \\
\hline 3 & & Corn-min & 830 \\
\hline 4 & & Corn & 237 \\
\hline 5 & & Grass-pasture & 483 \\
\hline 6 & & Grass-trees & 730 \\
\hline 7 & & Grass-pasture-mowed & 28 \\
\hline 8 & & Hay-windrowed & 478 \\
\hline 9 & & Oats & 20 \\
\hline 10 & & Soybean-notill & 972 \\
\hline 11 & & Soybean-mintill & 2455 \\
\hline 12 & & Soybean-clean & 593 \\
\hline 13 & & Wheat & 205 \\
\hline 14 & & Woods & 1265 \\
\hline 15 & & Buildings-Grass-Trees & 386 \\
\hline 16 & & Stone-Steel-Towers & 93 \\
\hline \multicolumn{3}{|r|}{ Total } & 10249 \\
\hline
\end{tabular}

spectral feature extraction. Therefore, all the input noise and training data are spectral vectors. First, the number of training data spectral bands in each dataset is reduced to 10 by PCA. The size of noise $z$ sent to the generator networks is $1 \times 1 \times 1$ and it is then converted to $10 \times 1 \times 1$ through the fractionally-stride convolution of generator $G$.Then the generated fake samples and true training samples are imported to the discriminator $D$ which can give the corresponding image labels $c$ and source labels $s$. The main architectures of the 1D-GANs and PCA-CNN for each dataset are shown in Table IV, and the n_class shown in Table IV represents the number of classes in each dataset

In order to have a fair comparison, the architecture of PCA-CNN is designed in such a way to be the same as discriminator $D$.The stride is 1 and the padding is set to 0 in this experiment. Similarly, we apply the same activation function as used in DCGAN. The batch normalization is also introduced to the networks for the specific layers. The batch normalization can stabilize training by normalizing the input for each unit to have zero mean and unit variance.

At the end of the common CNN, the fully connected neural nodes are activated by a linear function. Then, the corresponding labels are given through a softmax classifier. After the full connection and activation, the last layer neural nodes of discriminator $D$ are connected with both a softmax classifier and a sigmoid classifier. From the softmax classifier, one can obtain the predicted label $c$. From the sigmoid classifier, one can obtain the labels $s$ (fake or real) of the GAN.

In this experiment, our methods are compared with other feature extraction methods like the PCA, factor-analysis (FA) [46], locally linear embedding (LLE) [47], linear discriminant analysis (LDA) [48], independent component analysis (ICA) [49], PCA-CNN and recurrent neural network (RNN) [50]. FA 
TABLE IV THE ARCHITECTURES OF THE 1D-GAN

\begin{tabular}{c|cccccc}
\hline \hline Nets & NO. & Convolution & BN & Stride & Padding & $\begin{array}{c}\text { Activation } \\
\text { function }\end{array}$ \\
\hline \multirow{3}{*}{$G$} & 1 & $4 \times 1 \times 512$ & YES & 1 & 0 & ReLu \\
& 2 & $4 \times 1 \times 128$ & YES & 1 & 0 & ReLu \\
& 3 & $4 \times 1 \times 1$ & NO & 1 & 0 & Tanh \\
\hline \multirow{5}{*}{$D$} & 1 & $4 \times 1 \times 256$ & NO & 1 & 0 & LeakyReLu \\
& 2 & $4 \times 1 \times 512$ & YES & 1 & 0 & LeakyReLu \\
& 3 & $4 \times 1 \times 128$ & NO & 1 & 0 & NO \\
& 4 & $128 \times$ n_class & NO & - & - & Softmax \\
& & $128 \times 2$ & NO & - & - & Sigmoid \\
\hline \hline
\end{tabular}

TABLE V ClasSifiCATION RESUlts OBTAINED By DiFFERENT APPROACH ON SALINAS DATASET

\begin{tabular}{|c|c|c|c|c|c|c|c|c|c|c|}
\hline \multirow[t]{2}{*}{ Dataset } & \multirow{2}{*}{$\begin{array}{c}\text { Classifier } \\
\text { FE } \\
\text { methods }\end{array}$} & \multicolumn{9}{|c|}{$\mathrm{KNN}$} \\
\hline & & PCA & FA & LLE & LDA & ICA & $\mathrm{CNN}$ & PCA-CNN & RNN & 1D-GAN \\
\hline \multirow{20}{*}{ Salinas } & $\mathrm{OA}(\%)$ & 75.20 & 71.27 & 74.50 & 76.53 & 80.12 & 83.32 & 84.14 & 77.46 & 85.64 \\
\hline & (2) & \pm 1.42 & \pm 2.17 & \pm 1.58 & \pm 2.17 & \pm 1.90 & \pm 1.90 & \pm 2.17 & \pm 2.27 & \pm 2.96 \\
\hline & $\mathrm{AA}(\%)$ & 70.29 & 65.10 & 68.63 & 78.28 & 79.93 & 76.10 & 77.13 & 77.05 & 79.86 \\
\hline & (1) & \pm 0.98 & \pm 2.52 & \pm 1.36 & \pm 2.52 & \pm 3.20 & \pm 2.03 & \pm 2.52 & \pm 3.80 & \pm 3.19 \\
\hline & $K \succ 100$ & 72.14 & 68.20 & 70.13 & 72.82 & 77.68 & 81.34 & 83.67 & 74.81 & 83.67 \\
\hline & $\mathrm{N} \times 100$ & \pm 1.38 & \pm 2.48 & \pm 1.72 & \pm 1.38 & \pm 2.13 & \pm 1.42 & \pm 1.38 & \pm 2.51 & \pm 2.03 \\
\hline & Classifier & \multicolumn{9}{|c|}{ Logistic Regression } \\
\hline & $O A(\%)$ & 79.12 & 76.57 & 76.70 & 79.22 & 80.35 & 88.29 & 88.94 & 78.46 & 89.13 \\
\hline & $\mathrm{OA}(\mathrm{T})$ & \pm 0.98 & \pm 2.52 & \pm 1.76 & \pm 2.67 & \pm 2.23 & \pm 1.73 & \pm 2.17 & \pm 2.15 & \pm 2.19 \\
\hline & $\mathrm{AA}(\%)$ & 78.29 & 76.10 & 75.63 & 68.94 & 75.14 & 84.10 & 87.13 & 80.92 & 88.13 \\
\hline & ה & \pm 1.48 & \pm 2.52 & \pm 1.36 & \pm 3.47 & \pm 3.45 & \pm 2.03 & \pm 2.52 & \pm 2.61 & \pm 3.50 \\
\hline & $K \succ 100$ & 76.14 & 74.20 & 74.13 & 72.57 & 78.04 & 87.34 & 88.45 & 76.01 & 89.03 \\
\hline & $\mathrm{N} \times 100$ & \pm 1.72 & \pm 2.48 & \pm 1.72 & \pm 3.07 & \pm 2.09 & \pm 1.42 & \pm 1.38 & \pm 2.41 & \pm 2.62 \\
\hline & Classifier & \multicolumn{9}{|c|}{ RBF-SVM } \\
\hline & $\mathrm{OA}(\%)$ & 78.20 & 75.27 & 76.50 & 78.53 & 83.04 & 87.32 & 87.98 & 77.23 & 88.45 \\
\hline & (2) & \pm 2.42 & \pm 3.17 & \pm 2.58 & \pm 2.17 & \pm 2.17 & \pm 1.90 & \pm 2.17 & \pm 2.23 & \pm 2.80 \\
\hline & $\mathrm{AA}(\%)$ & 82.29 & 81.10 & 79.63 & 75.51 & 80.48 & 84.10 & 85.13 & 78.02 & 84.63 \\
\hline & $2 x+(10)$ & \pm 0.98 & \pm 3.52 & \pm 2.36 & \pm 2.52 & \pm 2.17 & \pm 2.03 & \pm 2.52 & \pm 3.82 & \pm 3.64 \\
\hline & $\mathrm{K} \times 100$ & 75.14 & 73.20 & 72.89 & 77.05 & 81.98 & 85.34 & 85.45 & 74.56 & 88.45 \\
\hline & Nרז0 & \pm 1.38 & \pm 3.48 & \pm 2.72 & \pm 1.38 & \pm 2.17 & \pm 1.72 & \pm 1.38 & \pm 2.41 & \pm 2.62 \\
\hline
\end{tabular}

is a linear statistical method designed for potential factors from observed variables to replace original data. LLE seeks a lower-dimensional projection of the data which preserves distances within local neighborhoods. LDA can be used to perform supervised dimensionality reduction, by projecting the input data to a linear subspace consisting of the directions which maximize the separation between classes. ICA separates a multivariate signal into additive subcomponents that are maximally independent. An RNN is a network which uses hidden layers or memory cells to learn features, and it adopts recurrent connections between neural activations at consecutive time steps. The effectiveness of different feature extraction methods is evaluated mainly through classification results. Additional classifiers such as the KNN classifier and SVM with a kernel like RBF-SVM for the evaluation.

In order to have a fair comparison, we use the grid search method to find the best parameters for these feature extraction methods. The results listed in Table V-VII are the best results obtained in the searching process. For the methods with the RBF-SVM classifiers, we use a "grid-search" [51] method to define the most appropriate $C$ and $\gamma$ [52]. In this manner, pairs of $(C, \gamma)$ are tried and the one with the best classification accuracy on the validation samples is picked. This method is convenient and straightforward. Moreover, the computational time to find the best parameters by grid-search is not much since there are only two parameters that need to be estimated. In this experiment, we do the search by exponentially growing sequences of $C$ and $\gamma$ like [23].

For PCA, FA, LDA and ICA, we select the number of features in the range of 10 to $N$ (i.e., the number of hyperspectral bands). The number of the neighbors in LLE is chosen from 1 to 15 and for KNN the range of the nearest neighbors varied between 1 and 10. Table V-VII shows the results obtained from the condition when the GAN models are 
TABLE VI CLASSIFICATION RESULTS OBTAINED BY DIFFERENT APPROACH ON KSC DATASET

\begin{tabular}{|c|c|c|c|c|c|c|c|c|c|c|}
\hline \multirow[t]{2}{*}{ Dataset } & \multirow{2}{*}{$\begin{array}{c}\text { Classifier } \\
\begin{array}{c}\mathrm{FE} \\
\text { methods }\end{array}\end{array}$} & \multicolumn{9}{|c|}{ KNN } \\
\hline & & PCA & FA & LLE & LDA & ICA & CNN & PCA-CNN & RNN & 1D-GAN \\
\hline \multirow{20}{*}{$\mathrm{KSC}$} & \multirow{2}{*}{$\mathrm{OA}(\%)$} & 68.20 & 67.27 & 69.50 & 76.61 & 76.70 & 83.32 & 84.14 & 78.26 & 86.14 \\
\hline & & \pm 3.42 & \pm 2.27 & \pm 1.87 & \pm 3.42 & \pm 2.72 & \pm 2.30 & \pm 3.42 & \pm 2.23 & \pm 3.80 \\
\hline & \multirow{2}{*}{$\mathrm{AA}(\%)$} & 58.29 & 54.10 & 59.63 & 64.02 & 66.23 & 78.10 & 79.13 & 68.10 & 79.58 \\
\hline & & \pm 4.28 & \pm 2.72 & \pm 1.71 & \pm 4.28 & \pm 3.42 & \pm 2.43 & \pm 4.28 & \pm 3.75 & \pm 3.21 \\
\hline & \multirow{2}{*}{$\mathrm{K} \times 100$} & 65.14 & 64.20 & 67.13 & 74.32 & 73.95 & 81.34 & 85.45 & 75.73 & 83.45 \\
\hline & & \pm 4.38 & \pm 2.07 & \pm 2.56 & \pm 2.63 & \pm 3.04 & \pm 2.63 & \pm 3.52 & \pm 2.67 & \pm 3.62 \\
\hline & Classifier & \multicolumn{9}{|c|}{ Logistic Regression } \\
\hline & \multirow{2}{*}{$\mathrm{OA}(\%)$} & 71.20 & 70.27 & 72.50 & 75.42 & 76.90 & 85.32 & 87.67 & 80.82 & 89.64 \\
\hline & & \pm 0.98 & \pm 2.52 & \pm 1.36 & \pm 2.35 & \pm 2.86 & \pm 2.03 & \pm 2.42 & \pm 1.99 & \pm 2.47 \\
\hline & \multirow{2}{*}{$\mathrm{AA}(\%)$} & 74.29 & 67.10 & 70.63 & 70.34 & 72.89 & 80.10 & 81.13 & 71.79 & 84.42 \\
\hline & & \pm 2.16 & \pm 2.52 & \pm 1.36 & \pm 3.71 & \pm 3.15 & \pm 2.03 & \pm 1.28 & \pm 2.87 & \pm 3.19 \\
\hline & \multirow{2}{*}{$\mathrm{K} \times 100$} & 70.14 & 68.20 & 71.13 & 71.96 & 74.00 & 83.34 & 85.15 & 78.63 & 88.23 \\
\hline & & \pm 2.38 & \pm 2.48 & \pm 1.72 & \pm 2.71 & \pm 2.05 & \pm 1.42 & \pm 1.52 & \pm 2.21 & \pm 2.62 \\
\hline & Classifier & \multicolumn{9}{|c|}{ RBF-SVM } \\
\hline & \multirow{2}{*}{$\mathrm{OA}(\%)$} & 70.20 & 69.27 & 69.50 & 71.61 & 78.21 & 86.32 & 86.14 & 80.30 & 88.16 \\
\hline & & \pm 2.10 & \pm 2.17 & \pm 1.58 & \pm 3.42 & \pm 2.97 & \pm 1.90 & \pm 1.42 & \pm 3.07 & \pm 2.80 \\
\hline & \multirow{2}{*}{$\mathrm{AA}(\%)$} & 60.29 & 59.10 & 60.63 & 66.13 & 74.65 & 81.80 & 81.02 & 70.66 & 83.13 \\
\hline & & \pm 2.98 & \pm 3.52 & \pm 1.36 & \pm 4.28 & \pm 2.45 & \pm 2.03 & \pm 1.28 & \pm 5.35 & \pm 3.54 \\
\hline & \multirow{2}{*}{$\mathrm{K} \times 100$} & 68.14 & 62.20 & 64.13 & 70.17 & 75.58 & 84.89 & 86.23 & 78.04 & 85.10 \\
\hline & & \pm 2.46 & \pm 2.15 & \pm 1.72 & \pm 2.63 & \pm 2.63 & \pm 1.52 & \pm 1.51 & \pm 3.53 & \pm 2.03 \\
\hline
\end{tabular}

TABLE VII CLASSIFICATION RESULTS OBTAINED BY DIFFERENT APPROACH ON INDIAN PINES DATASET

\begin{tabular}{|c|c|c|c|c|c|c|c|c|c|c|}
\hline \multirow[t]{2}{*}{ Dataset } & \multirow{2}{*}{$\begin{array}{c}\text { Classifier } \\
\begin{array}{c}\mathrm{FE} \\
\text { methods }\end{array}\end{array}$} & \multicolumn{9}{|c|}{$\mathrm{KNN}$} \\
\hline & & PCA & FA & LLE & LDA & ICA & $\mathrm{CNN}$ & PCA-CNN & RNN & 1D-GAN \\
\hline \multirow{20}{*}{$\begin{array}{l}\text { Indian } \\
\text { Pines }\end{array}$} & \multirow{2}{*}{$\mathrm{OA}(\%)$} & 48.20 & 47.27 & 47.50 & 55.52 & 57.60 & 61.32 & 63.14 & 53.61 & 64.39 \\
\hline & & \pm 5.42 & \pm 4.17 & \pm 3.58 & \pm 2.36 & \pm 1.21 & \pm 1.90 & \pm 1.52 & \pm 4.84 & \pm 2.46 \\
\hline & \multirow{2}{*}{$\mathrm{AA}(\%)$} & 40.29 & 40.10 & 39.63 & 40.34 & 41.80 & 55.10 & 58.89 & 37.57 & 56.13 \\
\hline & & \pm 5.98 & \pm 4.92 & \pm 3.36 & \pm 4.43 & \pm 2.82 & \pm 1.03 & \pm 1.42 & \pm 5.13 & \pm 3.19 \\
\hline & \multirow{2}{*}{$\mathrm{K} \times 100$} & 44.14 & 43.20 & 42.13 & 48.26 & 50.63 & 58.34 & 60.45 & 45.67 & 60.45 \\
\hline & & \pm 5.38 & \pm 4.48 & \pm 3.72 & \pm 2.61 & \pm 1.52 & \pm 1.42 & \pm 1.04 & \pm 5.52 & \pm 3.14 \\
\hline & Classifier & \multicolumn{9}{|c|}{ Logistic Regression } \\
\hline & \multirow{2}{*}{$\mathrm{OA}(\%)$} & 51.20 & 47.27 & 46.50 & 54.67 & 56.57 & 64.32 & 66.14 & 57.51 & 67.24 \\
\hline & & \pm 1.59 & \pm 2.23 & \pm 1.97 & \pm 1.98 & \pm 2.78 & \pm 1.47 & \pm 1.46 & \pm 2.53 & \pm 2.80 \\
\hline & \multirow{2}{*}{$\mathrm{AA}(\%)$} & 43.29 & 45.10 & 41.63 & 39.37 & 42.69 & 57.10 & 59.13 & 44.52 & 59.27 \\
\hline & & \pm 2.98 & \pm 3.52 & \pm 2.36 & \pm 2.76 & \pm 3.14 & \pm 3.03 & \pm 2.09 & \pm 4.03 & \pm 3.19 \\
\hline & \multirow{2}{*}{$\mathrm{K} \times 100$} & 49.14 & 46.20 & 43.13 & 49.05 & 51.39 & 61.16 & 64.10 & 51.24 & 63.45 \\
\hline & & \pm 1.68 & \pm 2.48 & \pm 1.28 & \pm 3.05 & \pm 3.01 & \pm 1.98 & \pm 1.52 & \pm 3.12 & \pm 2.62 \\
\hline & Classifier & \multicolumn{9}{|c|}{ RBF-SVM } \\
\hline & \multirow{2}{*}{$\mathrm{OA}(\%)$} & 50.20 & 43.27 & 44.50 & 53.65 & 58.47 & 65.32 & 67.32 & 53.66 & 68.64 \\
\hline & & \pm 1.42 & \pm 2.17 & \pm 1.58 & \pm 2.48 & \pm 2.14 & \pm 1.90 & \pm 2.09 & \pm 5.18 & \pm 2.14 \\
\hline & \multirow{2}{*}{$\mathrm{AA}(\%)$} & 47.29 & 38.10 & 40.63 & 47.34 & 52.48 & 60.10 & 62.13 & 39.17 & 64.45 \\
\hline & & \pm 0.98 & \pm 2.52 & \pm 1.36 & \pm 1.42 & \pm 2.98 & \pm 2.03 & \pm 1.90 & \pm 6.88 & \pm 3.25 \\
\hline & \multirow{2}{*}{$\mathrm{K} \times 100$} & 49.14 & 40.20 & 42.13 & 50.89 & 57.47 & 62.45 & 65.36 & 46.08 & 66.36 \\
\hline & & \pm 1.38 & \pm 2.48 & \pm 1.72 & \pm 2.13 & \pm 2.47 & \pm 1.42 & \pm 1.35 & \pm 7.02 & \pm 3.08 \\
\hline
\end{tabular}

trained using the 10 principal components of the original HSIs. Compared with the PCA, FA, LLE, LDA, ICA and RNN, the CNN-based methods show better performances in terms of accuracies, especially the 1D-GAN methods which all achieve the best accuracy on three different classifiers. Furthermore, these Tables illustrate that the classification results usually have a superior performance when feature extraction methods are followed by either LR or RBF-SVM classifiers. 
TABLE VIII CLASSIFICATION RESULTS OF 1D-GAN WITH DIFFERENT PRINCIPAL COMPONENTS ON THREE DATASETS

\begin{tabular}{c|c|ccccc}
\hline \hline Dataset & Method & PCA-3 & PCA-10 & PCA-50 & PCA-100 & PCA-All \\
\hline \multirow{5}{*}{ Salinas } & OA(\%) & $85.32 \pm 1.54$ & $89.13 \pm 2.19$ & $83.46 \pm 1.95$ & $83.32 \pm 1.32$ & $81.89 \pm 2.24$ \\
& AA(\%) & $89.15 \pm 0.39$ & $88.13 \pm 3.50$ & $74.39 \pm 1.47$ & $74.09 \pm 0.97$ & $73.14 \pm 3.10$ \\
& K×100 & $82.07 \pm 1.22$ & $90.45 \pm 2.62$ & $75.20 \pm 1.42$ & $76.60 \pm 1.03$ & $78.52 \pm 2.26$ \\
& Time(s) & 29.6 & 46.1 & 66.8 & 86.6 & 106.2 \\
\hline \multirow{6}{*}{ KSC } & OA(\%) & $76.89 \pm 2.24$ & $89.64 \pm 2.47$ & $82.74 \pm 1.89$ & $82.45 \pm 1.45$ & $82.33 \pm 1.34$ \\
& $\mathrm{AA}(\%)$ & $70.14 \pm 1.34$ & $84.13 \pm 3.19$ & $78.28 \pm 2.04$ & $77.28 \pm 2.04$ & $76.15 \pm 1.45$ \\
& $\mathrm{~K} \times 100$ & $74.52 \pm 1.26$ & $88.15 \pm 2.62$ & $83.41 \pm 1.23$ & $81.24 \pm 0.89$ & $81.48 \pm 1.26$ \\
& Time(s) & 19.5 & 32.7 & 68.2 & 142.4 & 178.5 \\
\hline \multirow{5}{*}{ Indian Pines } & $\mathrm{OA}(\%)$ & $64.09 \pm 3.27$ & $67.24 \pm 2.80$ & $64.35 \pm 2.09$ & $62.30 \pm 1.85$ & $59.58 \pm 2.56$ \\
& $\mathrm{AA}(\%)$ & $58.14 \pm 2.56$ & $59.27 \pm 3.19$ & $56.75 \pm 2.56$ & $53.75 \pm 1.56$ & $48.78 \pm 3.09$ \\
& $\mathrm{~K} \times 100$ & $61.32 \pm 2.21$ & $63.45 \pm 2.62$ & $61.53 \pm 1.03$ & $60.07 \pm 1.03$ & $57.00 \pm 2.13$ \\
& Time(s) & 23.2 & 37.2 & 85.4 & 115.6 & 153.4 \\
\hline \hline
\end{tabular}

For the Salinas dataset, 1D-GAN-LR exhibits the best OA, AA and $\mathrm{K}$, with improvements of $0.84 \%, 4.03 \%$ and 0.0169 over CNN-LR, respectively. Our approach outperforms 1D-GAN-KNN by $3.49 \%, 8.27 \%$ and 0.0536 in terms of OA, $\mathrm{AA}$ and $\mathrm{K}$, respectively. For the $\mathrm{KSC}$ data et, as can be seen, the OA of $1 \mathrm{D}-\mathrm{GAN}-\mathrm{LR}$ is $89.64 \%$, which is increased by $3.50 \%$ and $0.48 \%$ compared with KNN and RBF-SVM, respectively, it also increases $1.97 \%, 3.29 \%$ and 0.0308 in OA, AA, and $\mathrm{K}$ compared with 1D-PCA-CNN. For the Indian Pines dataset, the best performance is conducted by $1 \mathrm{D}-\mathrm{GAN}$ followed by RBF-SVM. In this context, 1D-GAN followed by RBF-SVM improves the OA, AA, and $\mathrm{K}$ of CNN-RBF-SVM by $3.32 \%$, $4.35 \%$, and 0.0391 , respectively. The results show that the 1D-GAN method gave the best performance in terms of OA, AA and Kappa for all three datasets.

Furthermore, detailed experiments with different principal components of 1D-GAN have been investigated to give a comprehensive comparison. Table VIII shows the classification results of $1 \mathrm{D}-\mathrm{GAN}$. There, the whitening principal component analysis (PCA) was utilized in the experiments, which is a modified PCA with identity covariance matrix. In the table, the PCA-3 PCA-10, PCA-50, PCA-100, and PCA-All represent the situations where we preserve three, 10, 50, 100, and all principal components, respectively.

In 1D-GAN, 10 principal components are used to condense the spectral information. Due to the fact that we only use spectral information in 1D-GAN, we try to preserve sufficient components (i.e., 10). Furthermore, compared with 3D-GAN, the computational complexity of $1 \mathrm{D}-\mathrm{GAN}$ is relatively low. We use relatively more components without high computation complexity. From Table VIII, one can see that the 10 principal components achieved the best classification accuracy on three datasets. If the number of the selected principal components is not sufficient, the classification results tend to be bad (e.g., when three are selected). Because of the dimensionality of the input is low (e.g., three PCs), one cannot formulate a deep network to capture the discriminant and nonlinear features efficiently. If the number of the selected principal components are too many (e.g., 100), the classification results tend to be bad too. In order to obtain a good classification performance, 10 principal components are selected in 1D-GAN. Because of the dimensionality of the input is too high (e.g., 100), it may cause the serious overfitting problem under the condition of limited training samples (i.e., 200).

\section{The classification results for $3 D-G A N$}

In 3D-GAN, the network considers both spectral and spatial features effectively, which can lead to a better performance in terms of classification accuracies than the ones obtained by 1D-CNN. As mentioned before, we preserve three principal components as the inputs of 3D-GAN. The architecture of the 3D-GAN are shown in Table IX. The number of classes for each dataset is represented by n_class. The networks $G$ and $D$ of 3D-GAN are deep CNNs with five convolution layers and proper activation functions. The discriminator $D$ is followed by a sigmoid classifier and a softmax classifier, which are used to classify the real/fake samples and the HSIs, respectively. The size of the input noise is $100 \times 1 \times 1$ and the generator converts the inputs to fake samples with the size of $64 \times 64 \times 3$. Then the generated samples are sent to the discriminator $D$. Bach normalization is used in some specific layers to boost the classification accuracy. In our practice, the model leads to instability if batch normalization is used in all layers.

The size of mini-batch was 100 and the learning rate was 0.0002. In this set of experiments, the number of training epochs for the CNNs and GANs is 600 .

The SVM-based and CNN-based five methods are included to give a comprehensive comparison. The classification results are shown in Tables X-XII. For the three datasets, we use $64 \times 64 \times n$ _bandwhich the n_band represents the number of spectral bands and $64 \times 64 \times 3$ neighbors of each pixel as the input 3D images in these methods without PCA and using PCA respectively. The input images are normalized into the range [-0.5 0.5$]$.

Due to the advantages of SVM, some SVM-based HSIs classifiers are included for comparison. The 3D-RBF-SVM (SVM with the radial basis function kernel) receives the 3D images as inputs. The extended morphological profile with 
TABLE IX THE ARCHITECTURES OF THE 3D-GAN

\begin{tabular}{c|cccccc}
\hline \hline \multirow{2}{*}{ Nets } & No. & Convolution & BN & Stride & Padding & $\begin{array}{c}\text { Activation } \\
\text { function }\end{array}$ \\
\hline \multirow{4}{*}{$G$} & 1 & $4 \times 4 \times 512$ & YES & 1 & 0 & ReLU \\
& 2 & $4 \times 4 \times 256$ & YES & 2 & 1 & ReLU \\
& 3 & $4 \times 4 \times 128$ & YES & 2 & 1 & ReLU \\
& 4 & $4 \times 4 \times 64$ & YES & 2 & 1 & ReLU \\
& 5 & $4 \times 4 \times 3$ & NO & 2 & 1 & Tanh \\
\hline \multirow{5}{*}{$D$} & 1 & $4 \times 4 \times 64$ & NO & 2 & 1 & LeakyReLU \\
& 2 & $4 \times 4 \times 128$ & YES & 2 & 1 & LeakyReLU \\
& 3 & $4 \times 4 \times 256$ & YES & 2 & 1 & LeakyReLU \\
& 4 & $4 \times 4 \times 512$ & YES & 2 & 1 & LeakyReLU \\
& 5 & $4 \times 4 \times 64$ & NO & 1 & 0 & NO \\
& 6 & $64 \times$ n_class & NO & - & - & Softmax \\
& $64 \times 2$ & NO & - & - & Sigmoid \\
\hline \hline
\end{tabular}

TABLE X CLASSIFICATION WITH SPECTRAL-SPatial FEATURES ON THE SALINAS DATASET

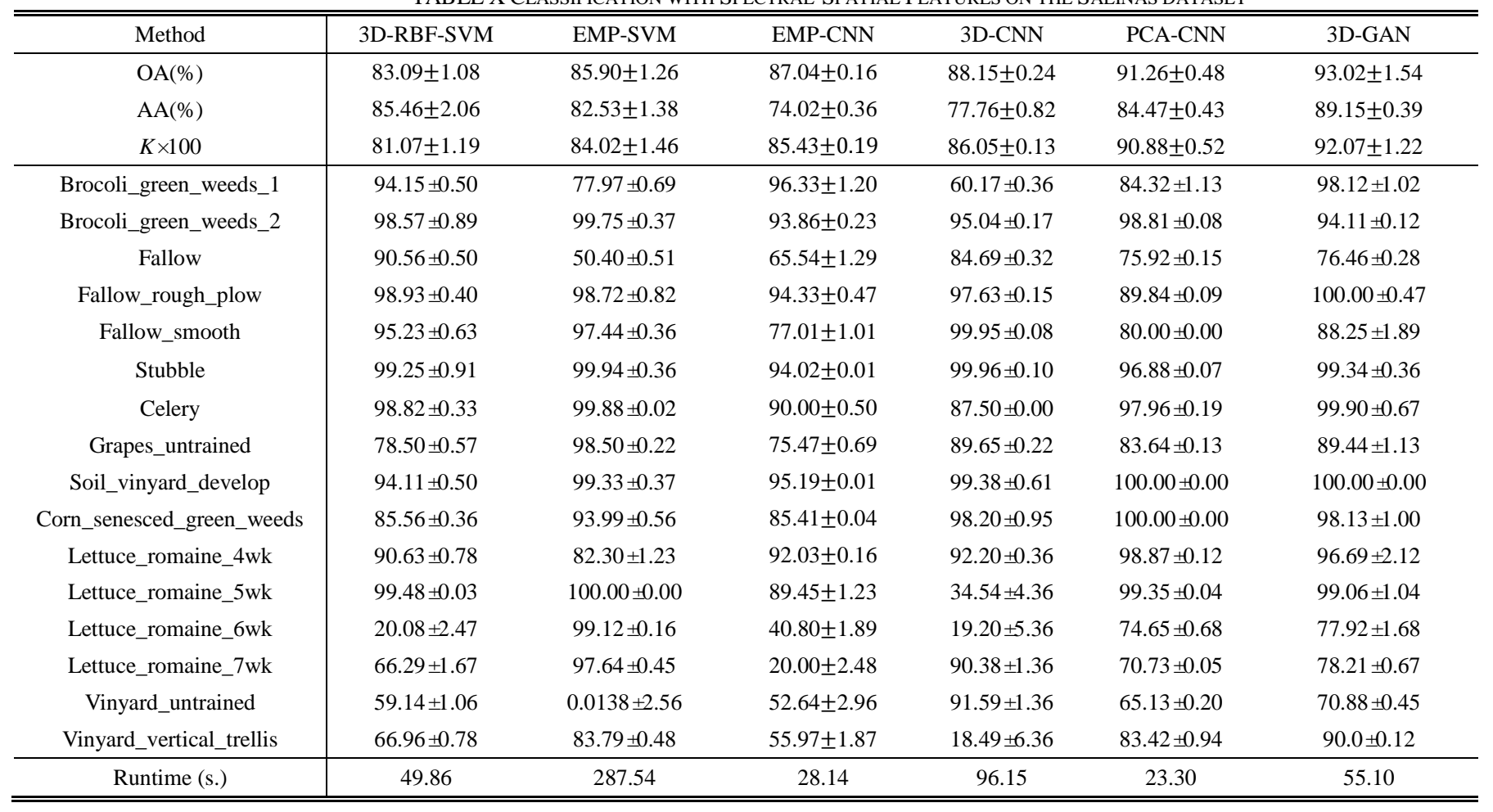

SVM (EMP-SVM) is a widely used spectral-spatial classifier for [5]. In the EMP-SVM method, three principal components from HSIs are computed and then the opening and closing operations are used to extract spatial information on the first three components. In the experiments, the shape structuring element (SE) is set as disk with an increasing size from 1 to 4 . Therefore, 24 spatial features are generated. The learned featured are fed to an RBF-SVM to obtain the final classification results.

Furthermore, 3D-CNN [21], which has the same architecture as the discriminator D in 3D-GAN, PCA-CNN, and EMP-CNN are also used for comparison. For the PCA-CNN, the CNN is conducted on the three principal components, which is useful when the training samples are limited [20]. From Tables X-XII, one can see that for Salinas dataset, the 3D-GAN exhibits the highest OA, AA and K, with an improvement of 1.76\%, 3.68\% and 0.0119 over PCA-CNN respectively. On the other hand, our 3D-GAN approach outperforms 3D-RBF-SVM by $9.93 \%$, $2.69 \%$ and 0.09 in terms of $\mathrm{OA}$, AA and $\mathrm{K}$, respectively. Furthermore, the proposed 3D-GAN obtains a better classification performance on Salinas dataset compared with the 3D-CNN and EMP-CNN. For the KSC and Indian Pines datasets, we can obtain the similar results. Compared with these state-of-the-art methods, the 3D-GAN demonstrates the best performance.

In term of the running time of different networks, the 3D-GAN needs a longer time to optimize a new network, which is caused by the update on both the generator network and 
TABLE XI ClASSIFICATION WITH SPECTRAL-SPATIAL FEATURES ON THE KSC DATASET

\begin{tabular}{c|cccccc}
\hline \hline Method & 3D-RBF-SVM & EMP- SVM & EMP-CNN & 3D-CNN & PCA-CNN & 3D-GAN \\
\hline OA(\%) & $76.13 \pm 0.24$ & $90.59 \pm 0.25$ & $96.94 \pm 0.13$ & $95.63 \pm 0.26$ & $96.02 \pm 0.42$ & $96.89 \pm 1.24$ \\
AA(\%) & $64.60 \pm 0.34$ & $85.83 \pm 0.28$ & $91.39 \pm 0.02$ & $89.65 \pm 0.21$ & $93.17 \pm 0.14$ & $94.14 \pm 0.40$ \\
K $\times 100$ & $73.52 \pm 1.30$ & $89.41 \pm 1.39$ & $96.60 \pm 0.14$ & $94.95 \pm 1.13$ & $95.27 \pm 0.49$ & $96.52 \pm 0.26$ \\
\hline Scrub & $88.68 \pm 0.69$ & $87.82 \pm 4.24$ & $90.14 \pm 0.89$ & $91.71 \pm 3.71$ & $96.189 \pm 0.56$ & $98.292 \pm 0.42$ \\
Willow swamp & $69.77 \pm 2.67$ & $80.14 \pm 7.06$ & $57.28 \pm 2.04$ & $89.73 \pm 10.1$ & $71.605 \pm 1.96$ & $79.835 \pm 1.45$ \\
CP hammock & $70.73 \pm 1.45$ & $87.64 \pm 6.19$ & $97.24 \pm 0.23$ & $92.16 \pm 5.33$ & $76.828 \pm 1.24$ & $98.438 \pm 0.14$ \\
Slash pine & $57.32 \pm 0.63$ & $86.64 \pm 4.79$ & $95.60 \pm 0.19$ & $86.94 \pm 6.27$ & $100.00 \pm 0.00$ & $86.508 \pm 1.12$ \\
Oak/Broadleaf & $42.85 \pm 2.56$ & $77.02 \pm 8.87$ & $63.75 \pm 1.56$ & $94.79 \pm 6.89$ & $100.00 \pm 0.00$ & $98.758 \pm 0.14$ \\
Hardwood & $32.24 \pm 0.62$ & $89.66 \pm 5.82$ & $92.07 \pm 1.03$ & $90.92 \pm 7.90$ & $96.943 \pm 0.78$ & $100.00 \pm 0.00$ \\
Swamp & $10.00 \pm 3.77$ & $83.37 \pm 17.8$ & $100.00 \pm 0.00$ & $91.57 \pm 6.14$ & $77.143 \pm 1.47$ & $97.143 \pm 1.06$ \\
Graminoid marsh & $42.23 \pm 3.64$ & $91.78 \pm 2.82$ & $100.00 \pm 0.00$ & $96.22 \pm 3.13$ & $79.814 \pm 1.89$ & $72.949 \pm 2.10$ \\
Spartina marsh & $84.00 \pm 0.25$ & $97.12 \pm 1.57$ & $91.37 \pm 0.56$ & $99.53 \pm 0.99$ & $100.00 \pm 0.00$ & $99.231 \pm 0.09$ \\
Cattail marsh & $81.88 \pm 1.35$ & $97.06 \pm 1.85$ & $99.56 \pm 0.01$ & $99.81 \pm 0.37$ & $99.752 \pm 0.04$ & $100.00 \pm 0.00$ \\
Salt marsh & $96.75 \pm 0.45$ & $99.64 \pm 1.12$ & $100.00 \pm 0.00$ & $99.79 \pm 0.30$ & $100.00 \pm 0.00$ & $100.00 \pm 0.00$ \\
Mud flats & $86.32 \pm 1.26$ & $99.24 \pm 2.11$ & $98.21 \pm 0.17$ & $97.69 \pm 2.31$ & $99.205 \pm 0.03$ & $96.481 \pm 1.23$ \\
Water & $100.00 \pm 0.00$ & $100.00 \pm 0.00$ & $100.00 \pm 0.00$ & $100.00 \pm 0.00$ & $100.000 \pm 0.00$ & $100.00 \pm 0.00$ \\
\hline Runtime (s.) & 47.94 & 292.78 & 27.68 & 57.53 & 24.56 & 48.57 \\
\hline \hline
\end{tabular}

TABLE XII CLASSIFICATION WITH SPECTRAL-SPATIAL FEATURES ON THE INDIAN PINES DATASET

\begin{tabular}{|c|c|c|c|c|c|c|}
\hline Method & 3D-RBF-SVM & EMP-SVM & EMP-CNN & $3 \mathrm{D}-\mathrm{CNN}$ & PCA-CNN & 3D-GAN \\
\hline $\mathrm{OA}(\%)$ & $58.01 \pm 1.08$ & $69.34 \pm 1.06$ & $86.48 \pm 0.13$ & $86.47 \pm 0.26$ & $87.27 \pm 1.01$ & $89.09 \pm 1.97$ \\
\hline $\mathrm{AA}(\%)$ & $50.56 \pm 2.06$ & $52.63 \pm 1.28$ & $68.19 \pm 0.26$ & $70.41 \pm 0.42$ & $80.17 \pm 0.48$ & $83.14 \pm 1.58$ \\
\hline$K \times 100$ & $52.07 \pm 1.19$ & $64.51 \pm 0.56$ & $84.23 \pm 0.16$ & $84.12 \pm 0.19$ & $85.24 \pm 1.29$ & $87.32 \pm 1.21$ \\
\hline Alfalfa & $77.35 \pm 1.50$ & $15.94 \pm 0.39$ & $10.43 \pm 1.78$ & $14.70 \pm 1.40$ & $15.00 \pm 1.34$ & $30.21 \pm 1.03$ \\
\hline Corn-notill & $18.52 \pm 1.06$ & $39.16 \pm 1.28$ & $86.71 \pm 1.25$ & $86.34 \pm 1.11$ & $86.98 \pm 0.96$ & $81.79 \pm 0.26$ \\
\hline Corn-mintill & $54.66 \pm 0.57$ & $70.75 \pm 0.59$ & $84.25 \pm 1.47$ & $89.49 \pm 0.70$ & $85.27 \pm 1.34$ & $75.93 \pm 1.26$ \\
\hline Corn & $32.30 \pm 0.39$ & $54.74 \pm 0.00$ & $76.92 \pm 2.69$ & $42.00 \pm 1.43$ & $94.09 \pm 1.63$ & $90.08 \pm 1.23$ \\
\hline Grass-pasture & $9.73 \pm 0.54$ & $68.37 \pm 0.47$ & $84.96 \pm 1.59$ & $85.91 \pm 0.23$ & $92.75 \pm 1.87$ & $86.39 \pm 2.12$ \\
\hline Grass-trees & $85.53 \pm 2.96$ & $96.21 \pm 1.16$ & $88.33 \pm 0.23$ & $92.75 \pm 0.30$ & $79.04 \pm 1.03$ & $93.28 \pm 0.23$ \\
\hline Grass-pasture-mowed & $10.27 \pm 1.36$ & $100.00 \pm 0.00$ & $10.67 \pm 2.35$ & $12.00 \pm 0.50$ & $53.57 \pm 1.65$ & $40.71 \pm 1.05$ \\
\hline Hay-windrowed & $78.50 \pm 0.23$ & $85.00 \pm 0.56$ & $100.00 \pm 0.00$ & $100.00 \pm 0.00$ & $91.59 \pm 0.48$ & $98.11 \pm 0.21$ \\
\hline Oats & $12.32 \pm 0.51$ & $15.78 \pm 1.19$ & $12.46 \pm 2.56$ & $10.00 \pm 1.20$ & $10.20 \pm 2.05$ & $20.00 \pm 1.96$ \\
\hline Soybean-notill & $62.28 \pm 3.36$ & $75.92 \pm 1.39$ & $89.85 \pm 0.45$ & $78.72 \pm 0.95$ & $73.25 \pm 1.14$ & $74.28 \pm 0.89$ \\
\hline Soybean-mintill & $66.86 \pm 1.61$ & $81.23 \pm 0.96$ & $91.55 \pm 1.04$ & $95.52 \pm 1.23$ & $90.30 \pm 0.78$ & $91.12 \pm 0.25$ \\
\hline Soybean-clean & $28.25 \pm 0.42$ & $31.85 \pm 0.51$ & $86.76 \pm 0.69$ & $89.47 \pm 0.39$ & $80.43 \pm 0.96$ & $84.99 \pm 1.46$ \\
\hline Wheat & $99.18 \pm 1.47$ & $98.23 \pm 0.28$ & $87.50 \pm 1.48$ & $80.00 \pm 0.00$ & $56.34 \pm 2.48$ & $49.75 \pm 2.45$ \\
\hline Woods & $85.98 \pm 1.28$ & $90.85 \pm 1.19$ & $88.37 \pm 1.67$ & $84.55 \pm 0.58$ & $91.62 \pm 0.69$ & $94.38 \pm 0.26$ \\
\hline Buildings-Grass-Trees & $13.82 \pm 0.35$ & $94.28 \pm 1.01$ & $53.53 \pm 2.48$ & $69.54 \pm 1.31$ & $74.58 \pm 0.78$ & $94.47 \pm 0.79$ \\
\hline Stone-Steel-Towers & $87.78 \pm 0.32$ & $95.24 \pm 0.67$ & $95.65 \pm 0.57$ & $89.34 \pm 1.08$ & $88.17 \pm 1.02$ & $88.22 \pm 1.16$ \\
\hline Runtime (s.) & 68.80 & 315.12 & 42.18 & 93.51 & 37.95 & 72.46 \\
\hline
\end{tabular}

discriminator network in one training epoch. Therefore, the computational complexity of GAN is approximately twice as much as the CNN-based methods, but our method possesses a superior ability in terms of classification accuracy.

Like 1D-GAN, detailed experiments with different principal components of 3D-GAN have been investigated to give a comprehensive comparison. Table XIII shows the classification results of 3D-GAN. In the experiment reported in the table, three principal components are investigated to obtain the main spectral information and their spatial neighborhood pixels are used to obtain the spatial information. Due to the fact that the spatial information is also included, we use relatively fewer components in the spectral domain compared with 1D-GAN.

From Table XIII one can observe that the PCA operation can improve the accuracy to some degree. In the experiment of 3D-GAN, we only chose three principal components because 
TABLE XIII ClassificAtion Results OF 3D-GAN with DifFERENT PRINCIPAL COMPONENTS ON Three Datasets

\begin{tabular}{c|c|ccccc}
\hline \hline Dataset & Method & PCA-3 & PCA-10 & PCA-50 & PCA-100 & PCA-All \\
\hline \multirow{5}{*}{ Salinas } & OA(\%) & $93.32 \pm 1.54$ & $95.12 \pm 0.75$ & $95.03 \pm 0.95$ & $94.53 \pm 1.02$ & $94.89 \pm 1.24$ \\
& AA(\%) & $89.15 \pm 0.39$ & $94.83 \pm 0.28$ & $94.39 \pm 0.47$ & $93.09 \pm 0.97$ & $93.14 \pm 0.40$ \\
& K×100 & $92.07 \pm 1.22$ & $94.41 \pm 1.39$ & $94.20 \pm 0.42$ & $93.60 \pm 0.45$ & $94.52 \pm 0.26$ \\
& Time(s) & 35.6 & 66.7 & 102.9 & 185.6 & 366.2 \\
\hline \multirow{5}{*}{ KSC } & OA(\%) & $96.89 \pm 1.24$ & $97.56 \pm 1.04$ & $97.14 \pm 0.89$ & $93.45 \pm 1.21$ & $93.33 \pm 1.34$ \\
& AA(\%) & $94.14 \pm 0.40$ & $92.14 \pm 1.06$ & $94.28 \pm 2.04$ & $89.28 \pm 2.04$ & $88.15 \pm 1.45$ \\
& K×100 & $96.52 \pm 0.26$ & $96.45 \pm 0.54$ & $95.41 \pm 0.23$ & $92.24 \pm 0.59$ & $91.48 \pm 1.26$ \\
& Time(s) & 25.05 & 49.5 & 108.1 & 200.4 & 330.8 \\
\hline \multirow{5}{*}{ Indian Pines } & OA(\%) & $89.09 \pm 1.97$ & $90.04 \pm 1.29$ & $88.97 \pm 1.87$ & $85.30 \pm 1.45$ & $83.58 \pm 1.12$ \\
& AA(\%) & $83.14 \pm 1.58$ & $85.02 \pm 1.54$ & $82.75 \pm 1.56$ & $81.75 \pm 1.56$ & $77.78 \pm 2.14$ \\
& K×100 & $87.32 \pm 1.21$ & $89.16 \pm 0.89$ & $86.53 \pm 1.03$ & $83.07 \pm 1.03$ & $79.00 \pm 1.63$ \\
& Time(s) & 40.2 & 60.7 & 167.9 & 348.6 & 532.7 \\
\hline \hline
\end{tabular}

TABLE XIV ClassificATION RESULtS BETWEEN NORMAL AND ISOLATED GAN NETWORKS ON THE THREE DATASETS

\begin{tabular}{c|ccc}
\hline \hline Dataset & & 3D-GAN & 3D-Isolated-GAN \\
\hline \multirow{3}{*}{ Salinas } & $\mathrm{OA}(\%)$ & $93.02 \pm 1.54$ & $90.09 \pm 0.56$ \\
& $\mathrm{AA}(\%)$ & $88.15 \pm 0.39$ & $85.76 \pm 1.31$ \\
& $K \times 100$ & $92.07 \pm 1.22$ & $88.05 \pm 0.96$ \\
\hline \multirow{3}{*}{ KSC } & $\mathrm{OA}(\%)$ & $97.69 \pm 0.24$ & $94.20 \pm 0.49$ \\
& $\mathrm{AA}(\%)$ & $94.14 \pm 0.40$ & $84.34 \pm 1.37$ \\
& $K \times 100$ & $96.52 \pm 0.26$ & $89.25 \pm 0.98$ \\
\hline \multirow{2}{*}{ Indian Pines } & $\mathrm{OA}(\%)$ & $90.69 \pm 0.86$ & $87.23 \pm 1.75$ \\
& $\mathrm{AA}(\%)$ & $83.14 \pm 1.82$ & $80.14 \pm 2.04$ \\
& $K \times 100$ & $89.62 \pm 0.26$ & $84.32 \pm 0.47$ \\
\hline \hline
\end{tabular}

the classification results are relatively good compared with other traditional methods, although the classification performance may be better if more components are selected. One should note that more principal components lead to a higher computational complexity and a longer training time. Furthermore, the classification accuracies descended when too many components (e.g., 100 components) were selected.

Taking the aforementioned factors into consideration, after performing the whiten PCA, we preserved three components in 3D-GAN.

To explore the effect of the adversarial component of GANs in classification, we also conduct experiments on all three datasets to see whether the classifier component of the 3D-GAN would perform better than for an isolated network of GANs. The isolated GAN network means that the discriminator $D$ updates its parameters in the training process while the generator $G$ does not update its parameters. In other words, the gradient flow from the discriminator $D$ does not broadcast to the generator $G$, and actually the two networks are not really in an adversarial manner. We call this training process 3D-Isolated-GAN and the results are listed in Table XIV.

In this experiment, 200 training samples are randomly chosen for each dataset, and from the Table we can see that the normal 3D-GAN outperforms the 3D-Isolated-GAN about $2.93 \%, 3.49 \%$, and $2.46 \%$ in terms of OA for the three datasets.
Therefore, we can make the conclusion that the adversarial action between two networks is highly important and influential in the training process.

\section{The visualization of adversarial samples and 3D-GAN}

\section{augmentation}

The GAN consists of a generator and a discriminator, which is a two-player minimax game between $G$ and $D$. If the discriminator cannot distinguish the real data from the synthetic fake data, we can conclude that the generated ability of $G$ has superior performance, and the whole adversarial network achieves the global optimality in theory [28].

The discriminator can be regarded as a classifier to get the classification results. On the other hand, the synthetic fake samples from the generator can be used to increase the number of training samples. The discriminator model is the one which directly accesses information in the dataset (e.g., the real samples from the distribution and the generator model learn based on error signals from the discriminator).

In the visualization experiment, the fake samples are generated by the generator (i.e., $G$ ) of 3D-GAN. Some selected fake and real samples on three hyperspectral datasets are shown in Fig. 8. In fact, it is difficult to distinguish the fake 


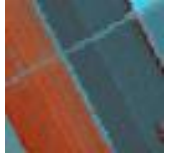

(a)

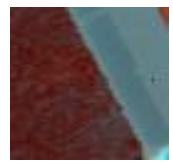

(b)

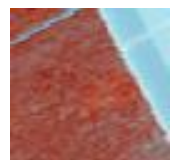

(c)

Label: Stubble

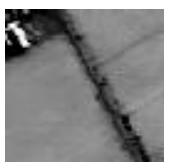

(a)

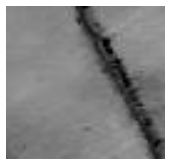

(b)

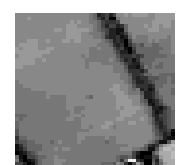

(c)

Label: Soil_vinyard_develop

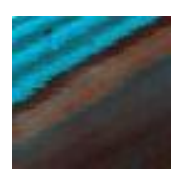

(a)

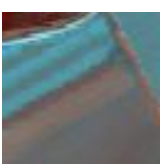

(b)

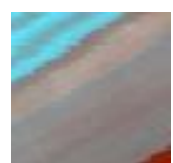

(c)

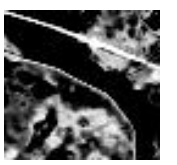

(a)

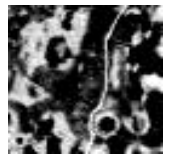

(a)

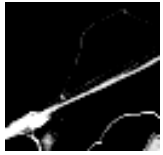

(a)

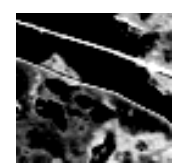

(b)

Label: Willow swamp

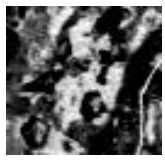

(b)

Label: Oak/Broadleaf

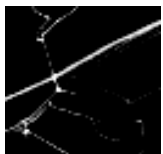

(b)

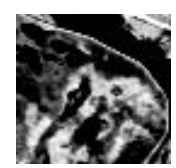

(c)

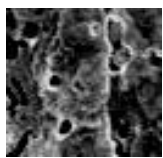

(c)

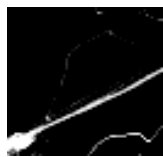

(c)
Label: Mud flats

(II)

(I)

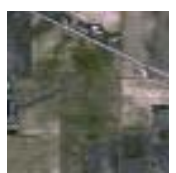

(a)

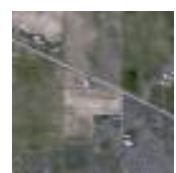

(b)

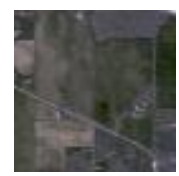

(c)

Label: Corn-notill

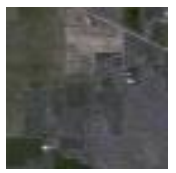

(a)

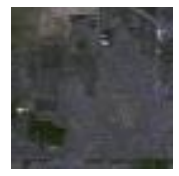

(b)

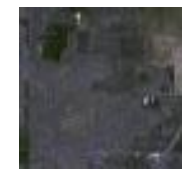

(c)

Label: Grass-trees

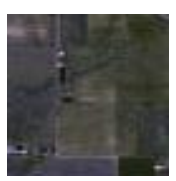

(a)

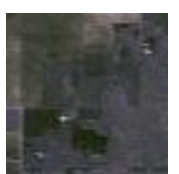

(b)

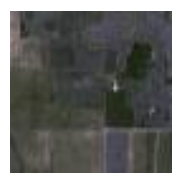

(c)

Label: Soybean-mintill

(III)

Fig. 8. The real data and generated fake data with same labels in different classes on three datasets: (I): Salinas data, (II) KSC dataset and (III) Indian Pines dataset, (a) real training data, (b) the first corresponding fake data and (c) the second corresponding fake data

samples from the real samples. From the Figure, one can see that at the end of the adversarial training, the generated samples get more and more details from the real data.

To verify the superior performance of the 3D-GAN augmentation methods, we feed the 100 fake samples, which are generated by 3D-GAN, into the training dataset. We suppose here that the original dataset has $N$ classes and that the fake samples can be endowed with the label $N+1$. Then, the real and fake samples are classified by the PCA-CNN and the 3D-GAN, which are called the Aug-PCA-CNN and
the3D-Aug-GAN, respectively. The classification results from these data augmentation methods and the original methods are shown in Tables XV-XVII. From the Tables, one can see that the OA of the methods after data augmentation on three dataset outperforms the methods without data augmentation. It is obvious that the additional fake samples can improve the classification performance compared with original methods.

\section{E. Classification Maps}

At last, in this subsection, the classification accuracies are 
TABLE XV ClassificATION WiTH DATA AUgMENTATION ON THE SALINAS DATASET

\begin{tabular}{|c|c|c|c|c|}
\hline Method & PCA-CNN & Aug-PCA-CNN & 3D-GAN & 3D-Aug-GAN \\
\hline $\mathrm{OA}(\%)$ & $91.26 \pm 0.48$ & $92.04 \pm 0.16$ & $93.02 \pm 1.54$ & $93.67 \pm 0.56$ \\
\hline $\mathrm{AA}(\%)$ & $84.47 \pm 0.43$ & $85.02 \pm 0.36$ & $88.15 \pm 0.39$ & $90.89 \pm 1.31$ \\
\hline$K \times 100$ & $90.88 \pm 0.52$ & $91.13 \pm 0.19$ & $92.07 \pm 1.22$ & $92.55 \pm 0.96$ \\
\hline Train time(s.) & 23.30 & 20.84 & 55.10 & 57.15 \\
\hline Test time(s.) & 26.45 & 26.63 & 26.56 & 26.12 \\
\hline
\end{tabular}

TABLE XVI CLASSIFICATION WITH DATA AUGMENTATION ON THE KSC DATASET

\begin{tabular}{c|cccc}
\hline \hline Method & PCA-CNN & Aug-PCA-CNN & 3D-GAN & 3D-Aug-GAN \\
\hline OA $(\%)$ & $96.02 \pm 0.42$ & $96.85 \pm 0.32$ & $97.69 \pm 0.24$ & $98.12 \pm 0.63$ \\
AA $(\%)$ & $93.17 \pm 0.14$ & $93.29 \pm 0.48$ & $94.14 \pm 0.40$ & $94.76 \pm 0.12$ \\
$K \times 100$ & $95.27 \pm 0.49$ & $95.71 \pm 0.56$ & $96.52 \pm 0.26$ & $98.05 \pm 0.20$ \\
\hline Train time(s.) & 24.56 & 19.96 & 48.57 & 2.75 \\
\hline Test time(s.) & 2.32 & 2.19 & 2.01 \\
\hline \hline
\end{tabular}

TABLE XVII CLASSIFICATION WITH DATA AUGMENTATION ON THE INDIAN PINES DATASET

\begin{tabular}{c|cccc}
\hline \hline Method & PCA-CNN & Aug-PCA-CNN & 3D-GAN & 3D-Aug-GAN \\
\hline OA $(\%)$ & $88.72 \pm 0.94$ & $89.34 \pm 1.45$ & $90.69 \pm 0.86$ \\
AA $(\%)$ & $80.17 \pm 0.48$ & $82.62 \pm 2.19$ & $83.14 \pm 1.82$ & $83.10 \pm 0.56$ \\
$K \times 100$ & $86.64 \pm 0.49$ & $87.43 \pm 1.98$ & $89.62 \pm 0.26$ & $89.95 \pm 0.13$ \\
\hline Train time(s.) & 37.95 & 41.04 & 57.46 & 5.46 \\
\hline Test time(s.) & 4.67 & 4.18 & 5.09 \\
\hline \hline
\end{tabular}

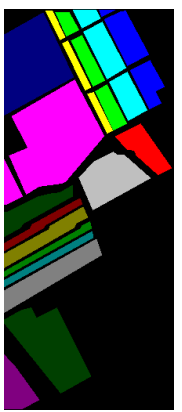

(a)

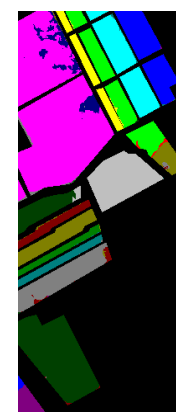

(b)

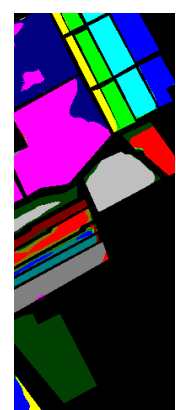

(c)

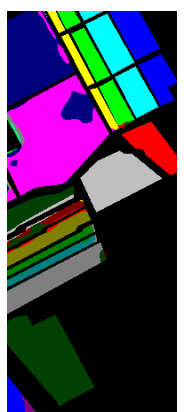

(d)

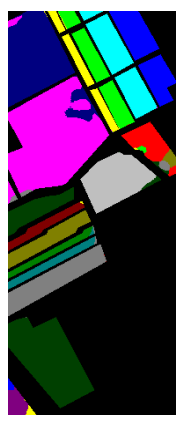

(e)

Fig. 9. Salinas: (a) Ground reference map, (b) to (e), classification maps for different classifiers: (b) EMP-SVM, (c) 3D-CNN, (d) 3D-GAN,(e) 3D-Aug-GAN

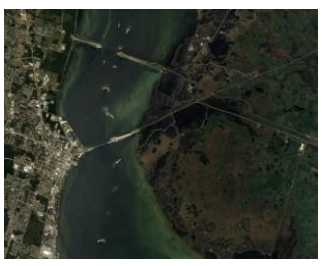

(a)

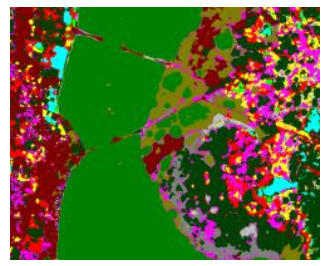

(b)

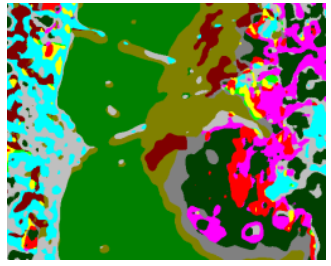

(c)

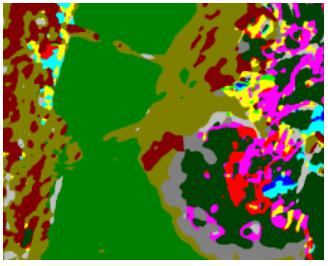

(d)

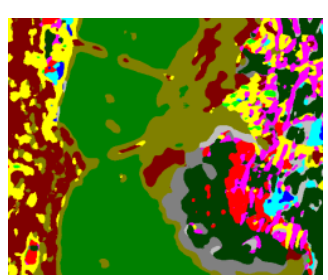

(e)

Fig. 10. KSC: (a) False color image, (b) to (e), classification maps for different classifiers: (b) EMP-SVM, (c) 3D-CNN, (d) 3D-GAN,(e) 3D-Aug-GAN

evaluated from a visual perspective. The methods including EMP-SVM, 3D-CNN, 3D-GAN and 3D-Aug-GAN with virtual samples are selected to classify the whole images. Figs. 9-11 are the classification maps for different methods on the three datasets. All parameters in these models are optimized. From Figs. 9-11, one can figure out how the different methods affect the classification results. The SVM-based methods always have the most errors for the three datasets (see Figs, 9(b), 10(b) and 11(b)). Especially for the Salinas dataset (Fig 9(b)), many pixels are misclassified at the top of the image with the SVM based method. By making the comparison between the true ground reference and the classification maps, one can see that the obtained classification results from CNNs and GANs are more precise, which show that the CNNs and GANs are 


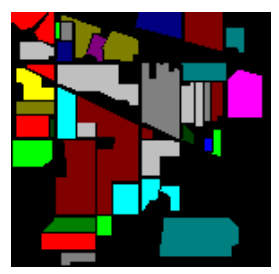

(a)

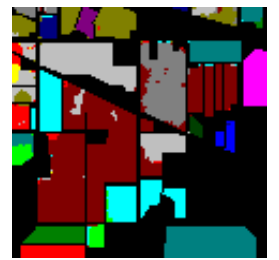

(b)

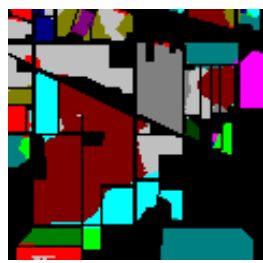

(c)

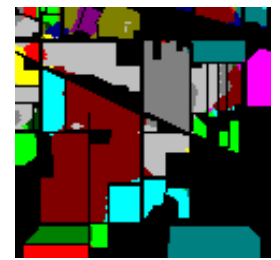

(d)

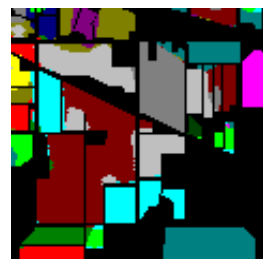

(e)

Fig. 11. Indian Pines: (a) Ground reference map, (b) to (e), classification maps for different classifiers: (b) EMP-SVM, (c) 3D-CNN, (d) 3D-GAN,(e) 3D-Aug-GAN

promising methods for HSI classification. Furthermore, the proposed GAN with data augmentation (3D-Aug-GAN) demonstrates an excellent visual classification performance, and it works pretty well under the condition of limited raining samples.

\section{CONCLUSION}

In this paper, the usefulness and effectiveness of GAN for HSIs classification are explored for the first time. With the help of GANs, the deep CNN achieves better performance in terms of classification accuracy compared with traditional CNN. Furthermore, the overfitting problem raised by CNNs is mitigated. In more detail, two frameworks are designed: (1) The first one, called the 1D-GAN, is based on spectral vectors and (2) the second one, called the 3D-GAN combines the spectral and spatial features. These two architectures demonstrated excellent abilities in feature extraction and image classification compared with other state-of-the-art methods. In the proposed GANs, PCA is used to reduce the high dimensionality of inputs, which is really important to stabilize the training procedure. Due to the large number of learnable parameters (e.g., weights) in deep models, deep CNNs suffer a lot with the problem of overfitting, while GAN can be regarded as a regularization technique which can mitigate the overfitting phenomenon in training process. Furthermore, the HSI samples that are generated by GAN are illustrated for the first time here, possibly opening a new window for generation of hyperspectral data generation. More importantly, generated adversarial samples are for the first time used as training samples for HSIs classification in this paper. Those samples significantly improve the classification performance. The aforementioned techniques show their huge potential of GANs for HSI classification.

\section{REFERENCES}

[1] C. Chang, "Hyperspectral Imaging: Techniques for Spectral Detection and Classification," in Plenum PublishingCo,N.Y. Dordrecht, The Netherlands: Kluwer, 2003.

[2] M. Fauvel, Y. Tarabalka, J. A. Benediktsson, J. Chanussot, and J. C. Tilton, "Advances in spectral-spatial classification of hyperspectral images", Proc. IEEE, vol. 101, no. 3, pp. 652-675, 2013.

[3] P. Ghamisi, J Plaza, Y Chen, J Li, and A. J. Plaza, "Advanced Spectral Classifiers for Hyperspectral Images: A review," IEEE Geosci. and Remote Sens. Mag., vol. 5, no. 1, pp.8-32, Mar. 2013.

[4] G. F. Hughes, "On the mean accuracy of statistical pattern recognizers," IEEE Trans. Inform. Theory, vol. IT-14, pp. 55-63, 1968.

[5] J. A. Gualtieri and R. F. Cromp, "Support vector machines for hyperspectral remote sensing classification," in Proc. SPIE 27th AIPR
Workshop, Washington, USA, Feb. 1998, pp. 221-232.

[6] F. Melgani and L. Bruzzone, "Classification of hyperspectral remote sensing images with support vector machines," IEEE Trans. Geosci. Remote Sens., vol. 42, no. 8, pp. 1778-1790, Aug. 2004.

[7] M. Fauvel, J. A. Benediktsson, J. Chanussot and J. R. Sveinsson, "Spectral and spatial classification of hyperspectral data using SVMs and morphological profiles," IEEE Trans. Geosci. Remote Sens., vol. 46, no. 11, pp. 3804-3814, Nov. 2008.

[8] P. Ghamisi, M. Mura, and J. A. Benediktsson, "A Survey on Spectral-Spatial Classification Techniques Based on Attribute Profiles," IEEE Trans. Geosci. Remote Sens., vol. 53, no. 5, pp.2335-2353, Nov. 2014.

[9] Y. Gu, J. Chanussot, X. Jia, J. A. Benediktsson, "Multiple Kernel Learning for Hyperspectral Image Classification: A Review," IEEE Trans. Geosci. Remote Sens., vol. 55, no. 11, pp. 6547-6565, Nov. 2017.

[10] Y. Yuan, L. Jianzhe, and W. Qi. "Hyperspectral Image Classification via Multitask Joint Sparse Representation and Stepwise MRF Optimization," IEEE Trans. Geosci. Remote Sens., vol. 46, no. 12, pp. 2966-2977, Oct. 2016.

[11] W. Qi, M. Zhaotie, L. Xuelong, "Locality Adaptive Discriminant Analysis for Spectral-Spatial Classification of Hyperspectral Images," IEEE Geosci. Remote Sens. Lett., vol. 14, no. 11, pp. 2077-2081, Sep. 2017.

[12] Y. Bengio, A. Courville, P Vincent, "Representation Learning: A Review and New Perspectives," IEEE Trans. on Pattern Anal. and Mach. Intell., vol. 35, no.8, pp. 1789-1828, Aug. 2013.

[13] G. Hinton, L.Deng, D.Yu, et al, "Deep Neural Networks for Acoustic Modeling in Speech Recognition: The Shared Views of Four Research Groups," IEEE Signal Process. Mag., vol. 29, no.6, pp. 82-97, Oct. 2012.

[14] K. Yao, G. Zweig, et al. "Recurrent Neural Networks for Language Understanding," In Proceeding of Interspeech. 2013.

[15] L. Zhang, L. Zhang, and B. Du. "Deep Learning for Remote Sensing Data: A Technical Tutorial on the State of the Art," IEEE Geosci. and Remote Sens. Mag., vol. 4, no. 2, pp. 22-40, Jun. 2016.

[16] Y. Chen, Z. Lin, X. Zhao, and G. Wang, "Deep learning-based classification of hyperspectral data," IEEE J. Sel. Topics Appl. Earth Observ. Remote Sens., vol. 7, no. 6, pp. 2094-2107, Jun. 2014.

[17] Y. Chen, X. Zhao, and X. Jia, "Spectral-spatial classification of hyperspectral data based on deep belief network," IEEE J. Sel. Topics Appl. Earth Observ. Remote Sens., vol. 8, no. 6, pp. 1-12, Jun. 2015.

[18] P. Zhong, Z. Gong, et al "Learning to Diversify Deep Belief Networks for Hyperspectral Image Classification," IEEE Trans. Geosci. Remote Sens., vol. 55, no. 6, pp. 3516-3530, Mar. 2017

[19] W. Hu, Y. Huang, L. Wei, F. Zhang, and H. Li, "Deep convolutional neural networks for hyperspectral image classification," J. of Sensors. 501,2015

[20] W. Li, G. Wu, et al. "Hyperspectral Image Classification Using Deep Pixel-Pair Features," IEEE Trans. Geosci. Remote Sens., vol. 55, no. 2, pp. 844-853, Nov. 2016.

[21] W. Zhao, S. Du, "Spectral-Spatial Feature Extraction for Hyperspectral Image Classification: A Dimension Reduction and Deep Learning Approach," IEEE Trans. Geosci. Remote Sens., vol. 54, no.8, pp. 4544-4554, Apr. 2016.

[22] J. Yue, W. Zhao, S. Mao, and H. Liu, "Spectral-spatial classification of hyperspectral images using deep convolutional neural networks," Remote Sens. Lett., vol. 6, no. 6, pp. 468-477, 2015.

[23] Y. Chen, H. Jiang, et al. "Deep Feature Extraction and Classification of Hyperspectral Images Based on Convolutional Neural Networks," IEEE Trans. Geosci. Remote Sens., vol. 54, no. 10, pp. 6232-6251, Oct. 2016.

[24] Y. Li, H. Zhang, and Q. Shen, "Spectral-spatial classification of 
hyperspectral imagery with 3D convolutional neural network," Remote Sens., vol. 9, no. 1, p. 67, 2017.

[25] H. Liang and Q. Li, "Hyperspectral imagery classification using sparse representations of convolutional neural network features," Remote Sens., vol. 8, no. 2, p. 99, 2016.

[26] Y. Chen, L. Zhu, et al, "Hyperspectral Images Classification with Gabor Filtering and Convolutional Neural Network," IEEE Geosci. Remote Sens. Lett., vol. 14, no. 12, pp. 2355 - 2359. Dec. 2017.

[27] V. Singhal, H. Aggarwal, et al "Discriminative Robust Deep Dictionary Learning for Hyperspectral Image Classification," IEEE Trans. Geosci. Remote Sens., vol. 55, no. 9, pp. 5274-5283. Jun. 2017.

[28] I. Goodfellow, J. Pouget-Abadie, M. Mirza, B. Xu, D. Warde-Farley, and Y. Bengio. "Generative adversarial nets," in NIPS 2014, Montréal, Canada, 2014, pp. 2672-2680.

[29] A. Radford, L. Metz, and S. Chintala, "Unsupervised Representation Learning with Deep Convolutional Generative Adversarial Networks," arXiv preprint arXiv: $1511.06434 \mathrm{v} 2,2016$.

[30] A. Creswell, T. White, V. Dumoulin, et al, "Generative Adversarial Networks: An Overview," IEEE Signal Process. Mag., Apr. 2017.

[31] M. Mirza, S. Osindero, "Conditional generative adversarial nets," arXiv preprint arXiv: 1411.1784, 2014.

[32] D. Pfau and O. Vinyals. "Connecting Generative Adversarial Networks and Actor-Critic Methods." arXiv preprint arXiv:1610.01945, 2017.

[33] G. Hinton and R. Salakhutdinov, "Reducing the dimensionality of data with neural networks," Science, vol. 313. no. 5786, pp. 504-507, Jul. 2006.

[34] I. Sutskever and G. E. Hinton, "Deep, narrow sigmoid belief networks are universal approximators," Neural Comput., vol. 20, no. 11, pp. 2629-2636, Nov. 2008.

[35] N. LeRoux and Y. Bengio, "Deep belief networks are compact universal approximators," Neural Comput., vol. 22, no. 8, pp. 2192-2207, Aug. 2010.

[36] Y. LeCun, L. Bottou, Y. Bengio, and P. Haffner, "Gradient-based learning applied to document recognition," Proc. IEEE, vol. 86, no. 11, pp. 2278-2324, Nov. 1998.

[37] N. Kruger, et al., "Deep hierarchies in primate visual cortex what can we learn for computer vision?" IEEE Trans. Pattern Anal. Mach. Intell., vol. 35, no. 8, pp. 1847-1871, Aug. 2013.

[38] A. Maas, A. HannuM, and A.Y. Ng. "Rectifier nonlinearities improve neural network acoustic models," in Proc. Int. Conf. Machine Learning (ICML), Atlanta, GA, USA, 2013.

[39] M. Arjovsky, S. Chintala, and L. Bottou, "Wasserstein GAN," arXiv preprint arXiv: 1701.07875, 2017.

[40] J. Zhu, T. Park, P. Isola, and A. A. Efros, "Unpaired Image-to-Image Translation using Cycle-Consistent Adversarial Networks", arXiv preprint arXiv: 1703.10593, 2017.

[41] A. Odena, C. Olan, and J. Shlens, "Semi-supervised learning with generative adversarial networks," arXiv preprint arXiv: 1606.01583, 2016.

[42] M. Mirza, S. Osindero, "Conditional generative adversarial nets," arXiv preprint arXiv: 1411.1784, 2014.

[43] A. Odena, C. Olah, and J. Shlens, "Conditional image synthesis with auxiliary classifier GANs," arXiv preprint arXiv:1610.09585, 2016.

[44] G. Licciardi, P. R. Marpu, J. Chanussot, and J. A. Benediktsson, "Linear versus nonlinear PCA for the classification of hyperspectral data based on the extended morphological profiles," IEEE Geosci. Remote Sens. Lett., vol. 9, no. 3, pp. 447-451, May. 2011.

[45] A. Krizhevsky, I. Sutskever, and G. E. Hinton. "ImageNet classification with deep convolutional neural networks," in NIPS, Lake Tahoe, USA, 2012, pp. 1097-1105.

[46] D. J. Bartholomew, F. Steele, J. Galbraith, and I. Moustaki, "Analysis of multivariate social science data," Struct. Equation Model. Multidisciplinary J., vol. 18, no. 4, pp. 686-693, Apr. 2011.

[47] H. Yang, F. Qin, and Y. Wang, "LLE-PLS nonlinear modeling method for near infrared spectroscopy and its application," Spectrosc. Spectral Anal., vol. 27, no. 10, pp. 1955-1958, Oct. 2007.

[48] W. Li, S. Prasad, J.E. Fowler, and L.M. Bruce, "Locality-Preserving Dimensionality Reduction and Classification for Hyperspectral Image Analysis", IEEE Trans. Geosci. Remote Sens., vol. 50, no. 4, pp.1185-1198, Apr. 2012.

[49] T. M. Tu, "Unsupervised signature extraction and separation in hyperspectral images: A noise-adjusted fast independent component analysis approach,” Opt. Eng., vol. 39, no. 4, pp. 897-906, Apr. 2000.

[50] L. Mou, P. Ghamisi, and X. Zhu, "Deep Recurrent Neural Networks for Hyperspectral Image Classification," IEEE Trans. Geosci. Remote Sens., vol. 55, no. 7, pp.3639-3655, Apr. 2017.

[51] J. Weston and C. Watkins, "Multiclass support vector machines," London: Department of Computer Science, University of London, 1998.

[52] C. Chang and C. Lin, "LIBSVM: A library for support vector machines," ACM Trans. Intell. Syst. Technol., vol. 2, no. 3, pp. 1-27, Mar. 2011.

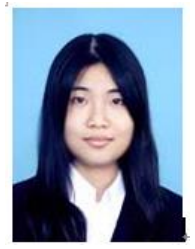

Lin Zhu has been working toward the Master's degree in the Department of Information Engineering, School of Electronics and Information Engineering, Harbin Institute of Technology, Harbin, China, since 2017.Her research interests include hyperspectral image classification, machine learning, and deep learning.

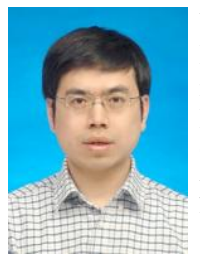

Yushi Chen (M'11) received the Ph.D. degree from Harbin Institute of Technology, Harbin, China, in 2008. Currently, he is an Associate Professor in the School of Electronics and Information Engineering, Harbin Institute of Technology, China. His research interests include remote sensing data processing and machine learning. For more details, please refer to http://homepage.hit.edu.cn/chenyushi.

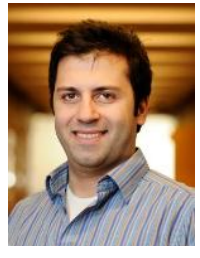

Pedram Ghamisi (S'12, M'15) is Research Scientist with the German Aerospace Center, Remote Sensing Technology Institute, Germany. His research interests include remote sensing and image analysis, with a special focus on the spectral and spatial techniques for hyperspectral image classification, multisensor data fusion, machine learning, and deep learning. For more info, please see http://pedram-ghamisi.com/.

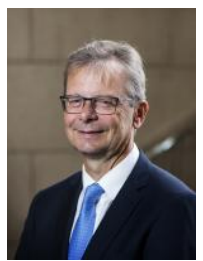

Jón Atli Benediktsson received the Cand.Sci. degree in electrical engineering from the University of Iceland, Reykjavik, in 1984, and the M.S.E.E. and Ph.D. degrees in electrical engineering from Purdue University, West Lafayette, IN, in 1987 and 1990, respectively. On July 1, 2015 he became the President and Rector of the University of Iceland. From 2009 to 2015 he was the Pro Rector of Science and Academic Affairs and Professor of Electrical and Computer Engineering at the University of Iceland. His research interests are in remote sensing, biomedical analysis of signals, pattern recognition, image processing, and signal processing, and he has published extensively in those fields. Prof. Benediktsson was the 2011-2012 President of the IEEE Geoscience and and Remote Sensing Society (GRSS) and has been on the GRSS AdCom since 2000. He was Editor in Chief of the IEEE Transactions on Geoscience and Remote Sensing (TGRS) from 2003 to 2008 and has served as Associate Editor of TGRS since 1999, the IEEE Geoscience and Remote Sensing Letters since 2003 and IEEE Access since 2013. He is on the Editorial Board of the Proceedings of the IEEE, the International Editorial Board of the International Journal of Image and Data Fusion, the Editorial Board of Remote Sensing, and was the Chairman of the Steering Committee of IEEE Journal of Selected Topics in Applied Earth Observations and Remote Sensing (J-STARS) 2007-2010. Prof. Benediktsson is a co-founder of the biomedical start up company Oxymap (www.oxymap.com). He is a Fellow of the IEEE and a Fellow of SPIE. Prof. Benediktsson was a member of the 2014 IEEE Fellow Committee. He received the Stevan J. Kristof Award from Purdue University in 1991 as outstanding graduate student in remote sensing. In 1997, Dr. Benediktsson was the recipient of the Icelandic Research Council's Outstanding Young Researcher Award, in 2000, he was granted the IEEE Third Millennium Medal, in 2004, he was a co-recipient of the University of Iceland's Technology Innovation Award, in 2006 he received the yearly research award from the Engineering Research Institute of the University of Iceland, and in 2007, he received the Outstanding Service Award from the IEEE Geoscience and Remote Sensing Society and the OECE Award from the School of ECE, Purdue University in 2016. He was co-recipient of the 2012 IEEE Transactions on Geoscience and Remote Sensing Paper Award and in 2013 he was co-recipient of the IEEE GRSS Highest Impact Paper Award. In 2013 he received the IEEE/VFI Electrical Engineer of the Year Award. In 2014 he was a co-recipient of the International Journal of Image and Data Fusion Best Paper Award. He is a member of the Association of Chartered Engineers in Iceland (VFI), Societas Scinetiarum Islandica and Tau Beta Pi. 